\title{
Scheduling isolated power systems considering electric vehicles and primary frequency response
}

\author{
Miguel Carrión ${ }^{\mathrm{a}, *}$, Ruth Domínguez ${ }^{\mathrm{a}}$, Miguel Cañas ${ }^{\mathrm{b}}$, Rafael Zárate-Miñano ${ }^{\mathrm{c}}$ \\ ${ }^{a}$ Department of Electrical Engineering, Universidad de Castilla - La Mancha, Toledo, Spain \\ ${ }^{b}$ Renewable Energy Research Institute and Department of Electrical Engineering, \\ Universidad de Castilla - La Mancha, Albacete, Spain \\ ${ }^{c}$ Department of Electrical Engineering, Universidad de Castilla - La Mancha, Almadén, Spain
}

\begin{abstract}
The incorporation of renewable energy sources in isolated power systems is being significantly slower than in well-connected power systems. The intermittency and uncertainty of the power output of most renewable power technologies prevent a greater usage of these technologies in isolated power systems, in which the supply security is the major concern. In this paper we formulate a stochastic unit commitment problem that allows the participation of electric vehicles in energy, reserve capacity and primary frequency response markets in order to increase the flexibility of the power system operation. We explicitly consider the uncertainty in the power demand and renewable power availability, as well as accounting for the possibilities of contingencies of generating units using a N-1 security criterion. The proposed formulation is tested on an actual isolated power system comprising 38 generating units and 8 buses.
\end{abstract}

Keywords:

Day-ahead scheduling, N-1 security criterion, reserve capacity, stochastic programming

\section{Introduction}

Global warming has become one of the major concerns in our society. As a consequence of this, the 2015 United Nations Climate Change Conference held in Paris was able to obtain an agreement of 174 countries calling for zero net anthropogenic greenhouse gas emissions to be reached during the second half of the $21^{\text {st }}$ century [1]. In this sense, regulators and governments have made significant efforts to incorporate renewable generation sources free of carbon emissions in power systems. For instance, [2] shows that wind and solar photovoltaic $(\mathrm{PV})$ power plants are the most installed generating technologies all around the world.

However, the integration of renewable units in isolated power systems has been generally smaller than in interconnected systems. Typically, isolated power systems are mainly fed by diesel generator units, which are very pollutant and involve high fuel costs. As an example, let us consider the isolated power systems of the Canary Islands, in Spain. Despite its optimal location in terms of availability of renewable sources, the installed capacity of wind and solar PV power units only represents $7 \%$ of the total. Opposite to this, these generation technologies represent up to $25 \%$ of the total capacity in the interconnected mainland Spanish power system [3]. The reason for this is that isolated systems are more vulnerable to unscheduled power fluctuations than interconnected power systems [4]. This prevents the installation of a large number of renewable units since their intermittent power output is more volatile and uncertain than that of conventional generation technologies.

\footnotetext{
*Corresponding author. Tel.: +34925 268800 (5750)

Email addresses: Miguel.Carrion@uclm.es (Miguel Carrión), Ruth.Dominguez@uclm.es (Ruth Domínguez), Miguel.Canas@uclm.es (Miguel Cañas), Rafael.Zarate@uclm.es (Rafael Zárate-Miñano)
} 
On the other hand, the usage of energy storage systems has been proven to be an effective tool to increase the flexibility of the power system operation [5], which may favor the integration of renewable power sources in isolated systems. In particular, electrochemical batteries present high charge and discharge efficiencies, long cycle life, and flexible power and energy characteristics that make them suitable for load shifting, mitigation of local load fluctuations and providing frequency regulation. In this way, the onboard batteries of plug-in electric vehicles (PEVs) can be used as energy storage systems when they are parked and connected to the grid. The capability vehicle-to-grid (V2G) allows the PEVs to inject the energy stored in their batteries into the distribution network [6]. This allows the electric vehicles to provide ancillary services, as reported in [7] and [8]. The economic viability of vehicle-to-grid operations has been analyzed in [9].

The incorporation of renewable energy sources in isolated power systems has been an active research topic in recent years. The authors in [10] propose a stochastic unit commitment for isolated power systems in which the risk of high operating costs is limited using the conditional value-at-risk risk measure. In [11] a unit commitment formulation that accounts for the requirements of spinning reserves is presented. The wind energy absorption capability in isolated systems is analyzed in [12]. In the same topic, [13] presents a novel procedure to calculate the maximum wind power penetration in an isolated power system that does not cause reliability problems. Reference [14] proposes a procedure to determine the optimal design of an isolated system with a high penetration of renewable energy sources. Finally, the usage of battery energy storages for frequency regulation in isolated power systems was studied in [15].

References [16]-[22] analyze the active participation of electric vehicles in the operation of power systems. In [16], several electric vehicle charging algorithms are proposed explicitly considering their negative impacts on the transmission and distribution grids. An agent-based coordinated dispatch strategy for electric vehicles and renewable units at distribution level is presented in [17]. In [18] a reserve contract optimization model designed for electric vehicles with vehicle-to-grid capability is proposed. In [19] the operation of the Dutch power system in 2020 is analyzed when the penetration of electric vehicles and renewable sources is expected to increase significantly. In the same vein, [20] investigates the optimal planning of the Nordic transmission system in 2050 for a $100 \%$ electric vehicle penetration. In [21] the provision of ancillary services in the power system of Singapore is analyzed, whereas [22] describes the practical operation of a fleet of electric vehicles with vehicle-to-grid capability.

The participation of electric vehicles in frequency regulation has been investigated in [23][30]. References [23] and [24] analyze the participation of electric vehicles in the primary frequency control aiming to maximize the integration of renewable power sources in isolated power systems. Reference [25] studies the contribution of electric vehicles to the primary frequency response in the Great Britain power system. Reference [26] presents a Markov decision problem that seeks to minimize the charging cost of a single electric vehicle that participates in the secondary frequency regulation. In [27] a decentralized control procedure for primary frequency regulation performed by PEVs is presented, whereas [28] proposes an adaptive droop procedure for electric vehicles to participate in the primary frequency regulation. In [29] the unidirectional real-time charging management problem faced by an electric vehicle aggregator in a market environment is formulated. A robust optimization framework is proposed in [30] for the formulation of a frequency regulation capacity scheduling problem in which a performancebased compensation scheme has been adopted. Reference [31] analyzes the real-time provision of primary response by a set of PEVs.

Considering the above, in this paper we propose a day-ahead energy and capacity scheduling model for isolated renewable-dominated power systems in which groups of PEV can participate as market agents. In this manner, the batteries of PEVs can be used as energy storages that participate in the day-ahead energy and spinning reserve capacity markets, as well as providing primary frequency response (PFR). It is expected that the participation of PEVs in the PFR will allow a reduction in the commitment of those generating units operating at a 
low capacity factor that aim to avoid large frequency variations if an unexpected unit failure occurs. To the best of the authors' knowledge, the formulation of this scheduling problem has not been investigated yet. This paper extends and improves the work presented by the authors in [33]. Unlike reference [33], in this paper unit commitment variables are considered to properly model contingencies of generating units and the provision of primary frequency response. We assume that dispatchable generating units equipped with synchronous generators and PEVs can participate in the primary frequency response. Instead of considering that PEVs are passive agents that are controlled by the independent system operator (ISO), the model proposed in this paper considers the active participation of PEVs in different trading floors. It is assumed that, under a smart grid framework, there exists a communication infrastructure that allows the efficient communication between PEVs and the system operator. Finally, an iterative solution procedure is proposed to solve the resulting mixed-integer linear programming problem that formulates the day-ahead scheduling considering the N-1 security criterion. The proposed procedure is tested on a realistic case study based on an actual isolated power system located in Spain.

\section{Model description}

In this section, the decision framework and the proposed optimization model are described. For the sake of clarity, the notation used throughout the paper is included Appendix A, whereas the detailed formulation of the model is provided in Appendix B.

\subsection{Decision framework}

We consider a power system formed by dispatchable and renewable power units, loads and electric vehicles. It is assumed that the power system has been designed to be able to integrate the charge of a significant number of PEVs. Renewable units are assumed to be non dispatchable with intermittent power output. Hereinafter, we will refer to these units as intermittent units. Generation offer and consumption bid curves are considered to be linear. We assume that the capacity of the loads to respond to pricing is very small and is therefore ignored. PEVs are categorized in different types, in such a way that PEVs with similar usage patterns are assigned to the same type. A PEV type is characterized by the set of hours in which PEVs are parked and connected to the grid. In this way, all PEVs belonging to the same type are assumed to have similar charging profiles. In order to account for the possibility of experiencing grid congestions, the transmission network is represented by the DC approximation [34].

Considering the power system described above, we propose a day-ahead market-clearing model that aims at determining:

- For each generating unit $g$ and each period $t$, the day-ahead energy output, $p_{g t}^{\mathrm{DA}}$, and the up and down reserve capacities, $c_{g t}^{\mathrm{up}}$ and $c_{g t}^{\mathrm{dw}}$, respectively.

- For each PEV group $k$ in each bus $n$ and period $t$, the energy sold and purchased in the day-ahead market, $p_{k n t}^{\mathrm{V}, \mathrm{DAD}}$ and $p_{k n t}^{\mathrm{V}, \mathrm{DAC}}$, the up and down reserve capacities, $c_{k n t}^{\mathrm{V} \text {,up }}$ and $c_{k n t}^{\mathrm{V}, \mathrm{dw}}$, and the PFR capacity $c_{k n t}^{\mathrm{V}, \mathrm{PR}}$. 
The scheduled up and down reserve capacities represent the maximum quantity of power that generating units and PEVs are forced to deploy upwards and downwards if an unexpected change either in the demand or in the renewable power output occurs. Therefore, this capacity is only dedicated to deploy the operating reserve used to follow hourly deviations of intermittent production and demand. In addition, we consider that dispatchable units and PEVs can provide PFR. Generating units provide PFR through a local characteristic droop in response to system frequency deviations from its nominal value [35]. In real power systems, all committed dispatchable generating units are usually forced to provide PFR and the remuneration for this service is settled outside of the market clearing procedure. In the case of PEVs, we consider that the response of PEVs to the variations in the frequency is determined by a droop control law [24]. We assume that the ISO compensates economically the availability of those PEVs willing to provide PFR. For that, PEVs submit in the day-ahead market clearing procedure the PFR capacity quantities and prices, $c_{k n t}^{\mathrm{V}, \mathrm{PR}}$ and $C_{k n t}^{\mathrm{V}, \mathrm{PR}}$, respectively. Note that PEV users can implicitly incorporate estimations about their battery degradation costs when bidding in the markets. In this manner, PEVs will only participate in the different trading floors if that participation is economically profitable for them. As an example, references [36] and [37] propose different procedures for determining the optimal bidding strategy of an aggrupation of PEVs participating in different day-ahead electricity markets. In particular, reference [37] explicitly considers the battery degradation costs for deciding the bidding strategy of PEVs. 


\section{Day ahead}
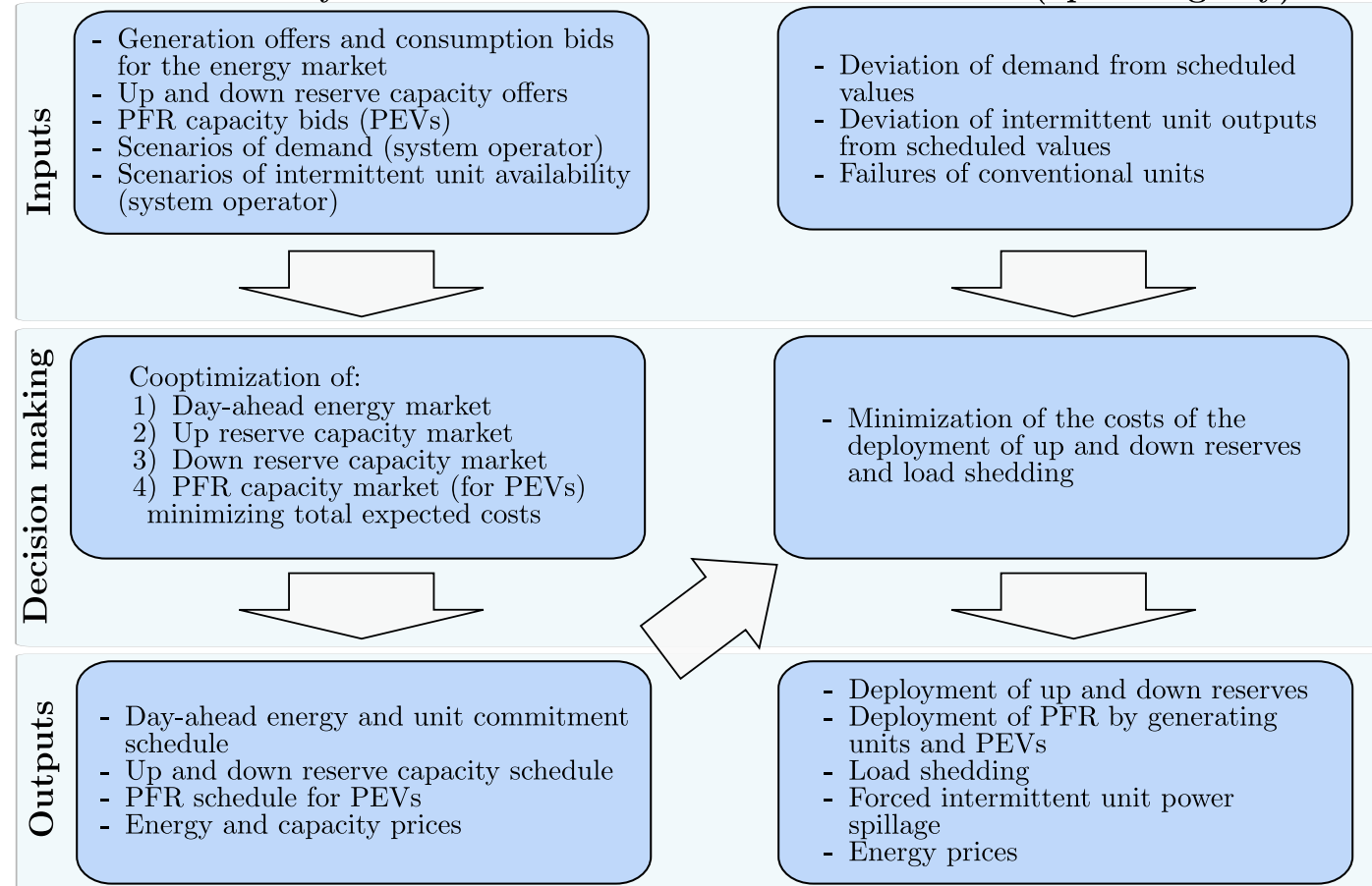

Figure 1: Decision-making flowchart

In this way, the proposed market-clearing procedure is formulated as a two-stage stochastic programming problem in which the first-stage represents the day-ahead market in which the scheduling of energy and reserve and PFR capacities are assigned to the different market agents. The real-time dispatch is characterized in the second-stage in which the deployment of the reserves is determined for a number of realizations of the uncertain parameters. From the point of view of the ISO, the main sources of uncertainty are the demand and the availability of the renewable power units, which are characterized using a set of scenarios. In addition, in order to consider the PFR in the day-ahead scheduling, a set of contingencies is considered in the real-time dispatch based on the N-1 security criterion. Therefore, all possible N-1 contingencies on generating units are considered at each time period and for each scenario. The objective function of this problem is the total expected operation cost. The flowchart represented in Figure 1 shows the decision-making described above.

The main advantage of co-optimizing simultaneously energy, reserve capacity and primary regulation markets is to obtain a unit commitment and day-ahead energy and capacity schedules flexible enough to cope with the different realizations of the uncertain parameters and generating unit failures, considering an explicit characterization of the uncertainty of the demand and the availability of intermittent and dispatchable power units.

It should be emphasized that the proposed day-ahead energy and capacity scheduling model 
is intended to be only used for assessing the energy, reserve capacity and primary regulation needs of the power system one day in advance. Then, based on the day-ahead energy and capacity scheduling obtained by the proposed model, adjustment and real-time markets must be cleared close to the instant when the energy is physically delivered using more detailed models of the transmission system and updated values of the demand and the availability of dispatchable and intermittent power units.

\subsection{Two-stage stochastic programming problem}

In this section we provide the description of the two-stage stochastic programming problem used to formulate the proposed scheduling model. For the sake of clarity, the main notation and the complete formulation of the model are provided in Appendix A and Appendix B, respectively. A general description of the objective function and the constraints of the model follows:

- The objective of the proposed model is to minimize the total operating cost, which comprises: i) the cost of scheduling energy and up, down, and PFR reserve capacity in the day-ahead market and the startup and shutdown costs of generating units, ii) the cost of the purchased and sold energy and up, down reserve and PFR capacities of PEVs in the day-ahead market, iii) the expected cost of deploying reserves to counteract the deviations between the day-ahead schedule and the real-time operation and the PFR.

- The first set of constraints of this model corresponds to the clearing of the day-ahead market. Related to the dispatchable generating units, we include the constraints that define the startup and shutdown costs and the ramping and capacity limits. A binary variable that represents the committed state of a unit is used to formulate those constraints. The power scheduled by intermittent units is limited to the available power predicted in the day ahead. Constraints imposing the limits of the transmission system are also included, as well as the power balance in the day-ahead market.

- The second set of constraints defines the real-time operation for the considered set of scenarios. We enforce the ramping, capacity and deployed reserve limits of the generating units. The power balance is defined considering the hourly deviations of the uncertain parameters, namely the intermittent production and the demand. The constraints related to the transmission system capacity are also included.

- The third set of constraints represents the primary frequency response. Those constraints determine the PFR according to the post-contingency state that results after the failure of a dispatchable power plant. The failure of a power plant is represented by a binary 
parameter, while the variation in the power output of the failed power plant is represented by a continuous variable. We impose that the power output to be generated by all committed units to supply that variation in the power output is bounded by either the droop of the unit or their capacity. On the contrary, the power output of the failed unit in the post-contingency state will be reduced up to zero. The participation of the PEVs in the PFR is limited by their droop and the power scheduled to this end in the day-ahead market. A limit on the frequency variation is imposed and the power balance for each post-contingency state is included.

- Finally, the constraints that define the state-of-charge of the PEVs in the real-time operation for each scenario and post-contingency state are considered. The limits on the stored energy and the charged/discharged power in each hour, scenario, and post-contingency state are enforced considering the purchased and sold energy and the reserve capacities scheduled in the day-ahead market.

The problem described above, here-in-after denoted as (P1), is a two-stage stochastic programming problem formulated as a mixed-integer linear programming problem, whose number of constraints and variables grows linearly with the number of considered scenarios and contingencies.

\section{Pricing}

In this section we propose a procedure for settling the financial remuneration that generating units and PEVs must receive or pay by their participation in the different trading floors described in Section 2.1. The pricing associated with the participation of the agents in the day-ahead and real-time energy markets is based on the procedure proposed in [32]. This pricing scheme is especially tailored for renewable-dominated power systems where the scheduling of energy and reserves is co-optimized. In this manner, as it is usual in most power systems, the energy traded in the day-ahead and real-time energy markets is priced using a locational marginal pricing mechanism, whereas the participation in reserve capacity and PFR markets is remunerated using a price-as-bid procedure. This pricing scheme is described below:

- In the day-ahead energy market, each generating unit located at node $n$ is paid for its scheduled energy in period $t$ at the scheduling energy price $\lambda_{n t}^{\mathrm{DA}}$. This price is equal to the dual variable associated with the energy balance constraint per bus and period enforced in the day-ahead energy market.

- In the day-ahead energy market, each PEV located at node $n$ is paid for its scheduled discharged energy in period $t$ at the scheduling energy price $\lambda_{n t}^{\mathrm{DA}}$. 
- In the day-ahead energy market, each PEV located at node $n$ is charged for its scheduled charged energy in period $t$ at the scheduling energy price $\lambda_{n t}^{\mathrm{DA}}$.

- In the reserve capacity market, each generating unit $g$ is paid for its scheduled up/down reserve capacity in period $t$ at the capacity cost offer $C_{g t}^{\text {cup }} / C_{g t}^{\text {cdw }}$.

- In the reserve capacity market, each PEV belonging to group $k$ in bus $n$ is paid for its scheduled up/down reserve capacity in period $t$ at the capacity cost offer $C_{k n t}^{\mathrm{V} \text {,cup }} / C_{k n t}^{\mathrm{V} \text {,cdw }}$.

- In the primary frequency regulation market, each PEV belonging to group $k$ in bus $n$ is paid for its scheduled PFR capacity in period $t$ at the capacity cost offer $C_{k n t}^{\mathrm{V}, \mathrm{PR}}$.

- In the real-time energy market, each generating unit located at node $n$ is paid/charged for its deployed up/down reserve in period $t$ and scenario $\omega$ at the real-time energy price $\frac{\lambda_{n t \omega}^{\mathrm{RT}}}{\pi_{\omega}}$. This price is equal to the dual variable associated with the energy balance constraint per bus, period and scenario enforced in the real-time energy market divided by the probability of scenario $\omega$.

- In the real-time energy market, each PEV located at node $n$ is paid/charged for its deployed up/down reserve in period $t$ and scenario $\omega$ at the real-time energy price $\frac{\lambda_{n t \omega}^{\mathrm{RT}}}{\pi_{\omega}}$.

Finally, note that the day-ahead and real-time prices are computed off-line after solving the scheduling model formulated in (P1). For doing so, the binary variables modeling the unit commitment of the generating units are fixed to their optimal values and a linear version of problem (P1) without binary variables is solved again. In this manner, it is possible to compute the dual variables associated with the energy balance constraints and finally obtain the dayahead and real-time prices, $\lambda_{n t}^{\mathrm{DA}}$ and $\frac{\lambda_{n t \omega}^{\mathrm{RT}}}{\pi_{\omega}}$.

\section{Solution Procedure}

Problem (P1) can be solved directly using commercial branch-and-cut solvers only for small instances. Due to the fact of considering contingencies associated with unit failures, if a large number of scenarios is considered, problem (P1) becomes intractable. However, observe that it is not necessary to consider all possible contingencies since most of them do not actually constraint the feasible region of problem (P1) [39]. In this manner there is a minimum set of contingencies over which it is sufficient to solve the original problem (P1) to achieve the same original solution of $(\mathrm{P} 1)$. In this paper we propose an iterative procedure to solve the original problem (P1) incorporating successively those contingencies that cause the greatest load shed in each iteration. The proposed procedure comprises the following steps: 
- Step 0: Initialization. We define an empty set of contingencies, $C^{0}=\varnothing$. We initialize the counter of iterations $\nu=1$ and the set of active contingencies $C_{1}=C^{0}$.

- Step 1: To solve (P1) considering only the active set of contingencies, $C_{\nu}$. Since we are considering a reduced number of contingencies, the optimal objective function of this problem constitutes a lower bound of problem $(\mathrm{P} 1), z_{\text {down }}^{\nu}$. Observe that this problem is easier to solve than the original problem (P1) since only a subset of contingencies is considered. The set of optimal first-stage variables of this problem is denoted by $\Theta_{\nu}^{\mathrm{FS}}$.

- Step 2: To solve (P1) considering the full set of contingencies $C$ and fixing the first-stage variables to the optimal values obtained in Step $1, \Theta_{\nu}^{\mathrm{FS}}$. Notice that this problem can be solved easily since first-stage variables are known. The optimal objective function of this problem constitutes an upper bound of problem (P1), $z_{\mathrm{up}}^{\nu}$.

- Step 3: Convergence checking. Check if the difference between the upper and lower bounds, $z_{\text {up }}^{\nu}-z_{\text {down }}^{\nu}$, is smaller than a specified tolerance. If so, the solution procedure stops and the solution of problem $(\mathrm{P} 1)$ is $z_{\text {up }}^{\nu}$ and the first-stage decision variables are $\Theta_{\nu}^{\mathrm{FS}}$. If not, the counter of iterations is increased, $\nu=\nu+1$, and the set of active contingencies is updated including in $C_{\nu}$ the contingency $c$ that causes the greatest load shed, i. e. $\left\{c: c=\operatorname{argmax}\left(\sum_{n, t, \omega} p_{n t w c}^{\mathrm{U}, \mathrm{PR}}\right) \mid c \in\left\{C-C_{\nu-1}\right\}\right\}$.

For the sake of clarity, the solution procedure described above is graphically represented in Figure 2 . 


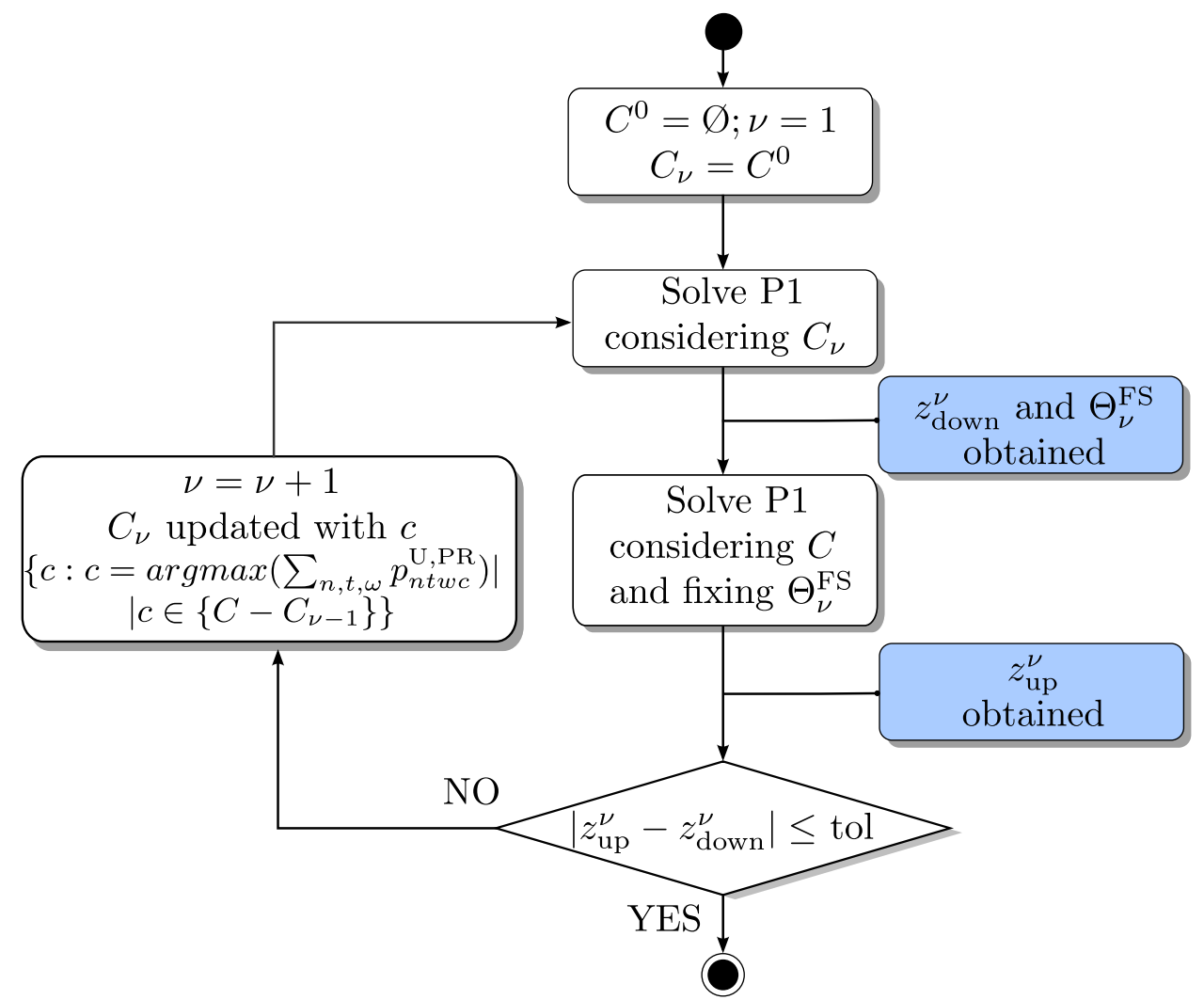

Figure 2: Solution procedure flowchart

\section{Numerical results}

A realistic case study based on the isolated power system of Lanzarote-Fuerteventura (LZFV) has been solved to analyze the performance of the proposed formulation. Lanzarote and Fuerteventura are two islands belonging to the Canary Islands (Spain) that are linked together by a submarine electricity interconnection [40]. These islands avail of a high estimated potential of wind and solar PV power, 170 and $500 \mathrm{MW}$, respectively [41]. Currently, these islands have together a population of around 250 thousand people with a maximum peak demand equal to $250 \mathrm{MW}$ in 2015. Additionally, the reduced area covered by these two islands, less than 2400 $\mathrm{km}^{2}$, minimizes the problem of the limited autonomy of current electric vehicles, which favors their usage.

\subsection{Input data}

The input data of the electric power system LZ-FV used in this case study is based on the power system described in [10]. This power system comprises 8 buses, 8 lines and 38 generating units. The generation mix consists of dispatchable (diesel, gas and mobile gas) and 
Table 1: Technical characteristics of dispatchable units.

\begin{tabular}{c|c|c|c|c|c|c}
\hline \hline \multirow{4}{*}{ Techn. } & Unit & Node & $\begin{array}{c}\text { Installed } \\
\text { capacity } \\
(\mathrm{MW})\end{array}$ & $\begin{array}{c}\text { Start-up } \\
\text { cost } \\
(€)\end{array}$ & $\begin{array}{c}\text { Operating } \\
\text { cost } \\
(€ / \text { MWh })\end{array}$ & $\begin{array}{c}\text { Minimum } \\
\text { power } \\
\text { output (MW) }\end{array}$ \\
\hline \multirow{5}{*}{ Diesel } & $1-3$ & 1 & 6.49 & 527 & 180.235 & 4.20 \\
\cline { 2 - 7 } & 4,5 & 1 & 12.85 & 1494 & 145.041 & 8.70 \\
\cline { 2 - 7 } & 6 & 1 & 20.51 & 2287 & 145.051 & 14.10 \\
\cline { 2 - 7 } & 7,8 & 1 & 17.20 & 1529 & 156.439 & 11.80 \\
\cline { 2 - 7 } & 11,12 & 6 & 3.82 & 270 & 174.018 & 11.80 \\
\cline { 2 - 7 } & 13 & 6 & 4.11 & 440 & 180.507 & 2.40 \\
\cline { 2 - 7 } & 14,15 & 6 & 6.21 & 227 & 180.805 & 4.20 \\
\cline { 2 - 7 } & 17 & 6 & 20.51 & 2287 & 145.510 & 14.09 \\
\cline { 2 - 7 } & 18,19 & 6 & 17.20 & 1529 & 156.439 & 11.80 \\
\cline { 2 - 7 } & 20 & 1 & 19.60 & 2468 & 292.051 & 6.79 \\
\cline { 2 - 7 } & 21 & 1 & 32.34 & 2468 & 245.011 & 6.79 \\
\cline { 2 - 7 } & 22 & 6 & 29.40 & 2255 & 251.703 & 9.69 \\
\hline \hline
\end{tabular}

intermittent (wind and solar PV) power plants. The technical characteristics of dispatchable and intermittent generating units are provided in Tables 1 and 2 , respectively. In order to attain a significant value of renewable generation, the renewable capacity per node installed in 2015 has been multiplied by 5. The startup and operating costs of wind and PV units, as well as their minimum power outputs, are assumed to be equal to zero. The shutdown cost of dispatchable units is 0.1 times their startup cost. The up and down reserve capacity costs of dispatchable units are considered to be identical and they are equal to 0.1 times the operating costs indicated in Table 1. In the same manner, up and down deployment costs of reserves are equal 1.1 and 0.85 times their operating costs, respectively. The frequency droop of thermal units is equal to $5 \%$. As is customary, we consider that renewable units do not participate in the reserve capacity and frequency regulation services. For simplicity, the damping factor of the load has been neglected. This assumption may lead to the overestimation of the frequency regulation needs of the system. The forced outage rate of thermal units is $3 \%$. The cost of unserved demand is fixed to $€ 10000 / \mathrm{MWh}$.

Due to limitation of space, we refer the interested reader to [10] for further information on the transmission network data and topology of the LZ-FV power system.

We consider that a number of electric vehicles with charging point in buses 3 and 6 actively participate in the electricity market operation. These electric vehicles represent $20 \%$ of all vehicles in each of these buses. Electric vehicles are grouped into three sets that have been defined based on the mobility study [42]. The description of the characteristics of each group is included in Table 3. This table describes the time interval in which vehicles are connected to the grid as well as the relative and absolute numbers of PEVs in each group. 
Table 2: Technical characteristics of intermittent units.

\begin{tabular}{c|c|c|c}
\hline \hline \multirow{4}{*}{ Techn. } & Unit & Node & $\begin{array}{c}\text { Installed } \\
\text { capacity } \\
(\mathrm{MW})\end{array}$ \\
\hline \multirow{4}{*}{ Wind } & 25 & 1 & 38.250 \\
\cline { 2 - 4 } & 26 & 2 & 5.625 \\
\cline { 2 - 4 } & 27 & 5 & 8.500 \\
\cline { 2 - 4 } & 28 & 8 & 5.625 \\
\cline { 2 - 4 } & 29 & 8 & 7.695 \\
\hline \multirow{5}{*}{ PV } & 30 & 1 & 7.115 \\
\cline { 2 - 4 } & 31 & 2 & 8.160 \\
\cline { 2 - 4 } & 32 & 3 & 1.530 \\
\cline { 2 - 4 } & 33 & 4 & 2.040 \\
\cline { 2 - 4 } & 35 & 5 & 1.555 \\
\cline { 2 - 4 } & 36 & 6 & 18.870 \\
\cline { 2 - 4 } & 37 & 7 & 7.225 \\
\cline { 2 - 4 } & 38 & 8 & 3.060 \\
\hline \hline
\end{tabular}

Table 3: PEV type characterization

\begin{tabular}{cccccc}
\hline \hline Type & $\begin{array}{c}\text { Beginning } \\
\text { hour }\end{array}$ & $\begin{array}{c}\text { Ending } \\
\text { hour }\end{array}$ & Duration & $\begin{array}{c}\text { Percentage } \\
\text { of PEVs }\end{array}$ & $\begin{array}{c}\text { Number } \\
\text { of PEVs }\end{array}$ \\
\hline 1 & 17.00 & 8.00 (next day) & 15 hours & $50 \%$ & 5151 \\
2 & 20.00 & 8.00 (next day) & 12 hours & $35 \%$ & 3606 \\
3 & 8.00 & 17.00 & 9 hours & $15 \%$ & 1545 \\
\hline \hline
\end{tabular}

Each electric vehicle is equipped with a $40 \mathrm{kWh}$ battery and it is considered that the daily distance driven for each vehicle is $35 \mathrm{~km}$ [33]. The energy consumption is $0.196 \mathrm{kWh} / \mathrm{km}$. The efficiency of charging and discharging the batteries is equal to 0.88. Electric vehicles are charged under a charging voltage of $400 \mathrm{~V}$ and a peak power transfer rate of $11 \mathrm{~kW}$. These values are based on the technical specifications of the Renault Zoe [43]. The specified frequency droop of PEVs is $11 \mathrm{~kW} / \mathrm{mHz}[23]$ and the maximum duration of PFR is 15 minutes.

Consumption and generation bids and offers of electric vehicles in the day-ahead energy market are $€ 350 / \mathrm{MWh}$ and $€ 460 / \mathrm{MWh}$, respectively. The reserve capacity costs are 0.02 times the energy offers in the day-ahead market. The deployment costs of up and down reserves are equal to 1.05 and 0.95 times the generation and consumption bid costs in the day-ahead market, respectively. The considered PFR capacity costs are $€ 5 / \mathrm{MW}$.

The solar PV and wind power availabilities and the system demand are characterized in LZ and FV as stochastic variables and they are modeled using a set of scenarios. Table 4 provides the ARIMA models that are proposed to characterize the demand, wind speed and solar radiation during 2015 in the LZ-FV power system. Historical data of demand are obtained from the Spanish system operator [3]. Wind speeds and solar radiation in each island are obtained from the Spanish meteorological agency [44]. The power curve of a $2 \mathrm{MW}$ wind turbine (Vestas V80/2000 with a hub height of $80 \mathrm{~m}$ ) is used to generate wind power production scenarios. The 
power curve of this turbine model can be found in [45].

Table 4: Parameters of the ARIMA models of demand, wind speed and global solar radiation

\begin{tabular}{ccc}
\hline \hline & Demand (MW) & $\phi_{1}=0.8487, \phi_{24}=0.9623, \phi_{48}=0.6114$ \\
& $\operatorname{ARIMA}(1,0,1) \times(2,1,2)_{24}$ & $\theta_{1}=-0.3607, \theta_{24}=1.5353, \theta_{48}=-0.7222$ \\
\cline { 2 - 3 } LZ & Wind speed $(\mathrm{m} / \mathrm{s})$ & $\phi_{1}=0.9615, \phi_{24}=0.9955$ \\
& $\theta_{1}=0.4135, \theta_{24}=0.9348$ \\
\cline { 2 - 3 } & ARIMA $(1,0,1) \times(1,0,1)_{24}$ & $\phi_{1}=1.1336, \phi_{2}=-0.3790, \phi_{24}=0.1135$ \\
& Solar radiation $\left(\mathrm{W} / \mathrm{m}^{2}\right)$ & $\theta_{1}=0.3879, \theta_{24}=0.9402$ \\
\hline \multirow{2}{*}{ FV } & $\phi_{1}=0.8466, \phi_{24}=-0.7137, \phi_{48}=0.2448$ \\
& Demand $(2,0,1) \times(1,1,1)_{24}$ & $\phi_{1}=0.9883, \phi_{24}=0.3138$ \\
& ARIMA $(1,0,1) \times(2,1,2)_{24}$ & $\theta_{1}=-0.3382, \theta_{24}=-0.0332, \theta_{48}=0.8763$ \\
\cline { 2 - 3 } & ARIMA $(1,0,1) \times(1,0,1)_{24}$ & $\theta_{1}=0.2215, \theta_{24}=0.1354$ \\
\cline { 2 - 3 } & Solar radiation $\left(\mathrm{W} / \mathrm{m}^{2}\right)$ & $\phi_{1}=1.1336, \phi_{2}=-0.3790, \phi_{24}=0.1135$ \\
& ARIMA $(2,0,1) \times(1,1,1)_{24}$ & $\theta_{1}=0.3879, \theta_{24}=0.9402$ \\
\hline \hline
\end{tabular}

The dependency between stochastic processes in the locations of LZ and FV is modeled considering the quasi-contemporaneous correlation of the series of errors [46].

An initial set of 200 scenarios is considered, which has been afterwards reduced to a final set of 12 scenarios by means of the scenario reduction algorithm presented in [47]. This algorithm iteratively selects the scenarios that minimize the probability distance between the original set of scenarios and the reduced one. This algorithm also assigns a probability for each selected scenario. Our numerical experiments with different numbers of scenarios prove that 12 scenarios are enough to ensure tractability and numerical stability in the optimal variables. As the analysis of scenario generation and reduction techniques is not the objective of this paper, due to limitation of space, we do not provide these results in this case study.

\subsection{Results}

The input data described in the previous subsection have been applied to two different cases. The first one considers a planning horizon of a single day. This case will allow us to test in detail the performance of the proposed formulation. The second case spans a full year and will enable us to analyze the active participation of electric vehicles in a comparatively larger planning horizon. In this case, a sensitivity analysis with respect to the installed renewable capacity and the participation of PEVs in different trading floors is performed. All simulations are performed with CPLEX 12.6.1 using a server with four 3.0 GHz processors and $250 \mathrm{~GB}$ of RAM. The optimality gap is set to $0.01 \%$.

\section{Case 1). Single-day simulation}

In this case a planning horizon of 24 hours is analyzed corresponding to February 24th of 2015 in the LZ-FV power system. Figure 3 shows the aggregated demand, wind and solar power 
scenarios generated using the ARIMA models provided in Table 4.
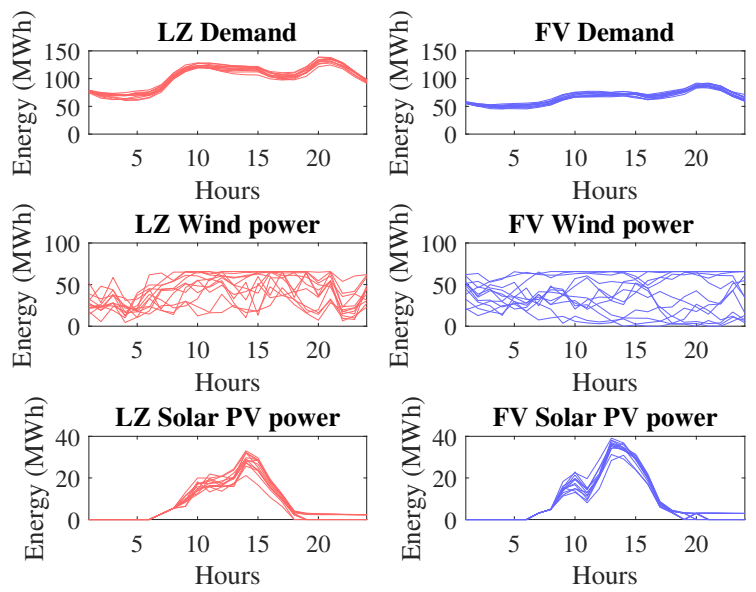

Figure 3: Demand, wind and solar power scenarios

The resulting operating cost for the considered day is equal to $435.9 \mathrm{k} €$, which is obtained after 3 iterations. The total solution time is 355 seconds. Figure 4 shows the upper and lower bounds obtained in each iteration. It is noted that the difference between both bounds is practically null in the second iteration.

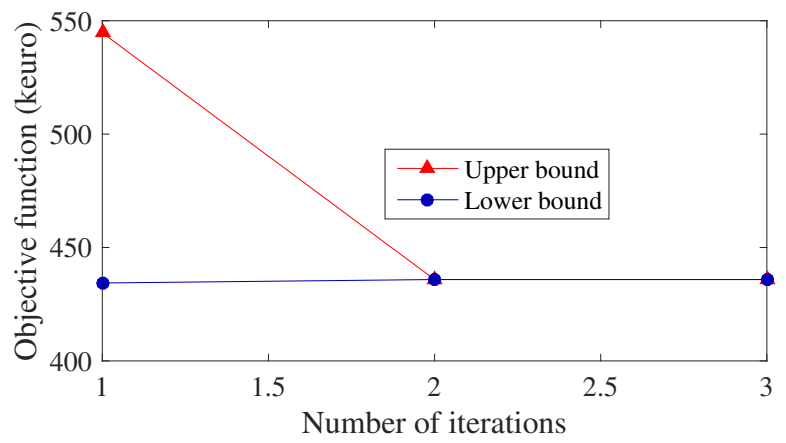

Figure 4: Upper and lower bound of the objective function in each iteration

The resulting day-ahead energy and reserve capacity schedules are depicted in Figures 5a and 5b. Observe that the color code is the same for both figures. Negative values of energy in the day-ahead energy market represented in Figure 5a correspond to the charging of PEVs. For the sake of clarity, up and down reserve capacities are represented using positive and negative values in Figure 5b, respectively. These figures show that diesel units supply most of the demand in the day-ahead energy market. PEVs are mainly charged during off-peak demand periods at the beginning and at the end of the day. PEVs also contribute to the provision of up and down reserve capacity in a lesser extent than diesel units. 


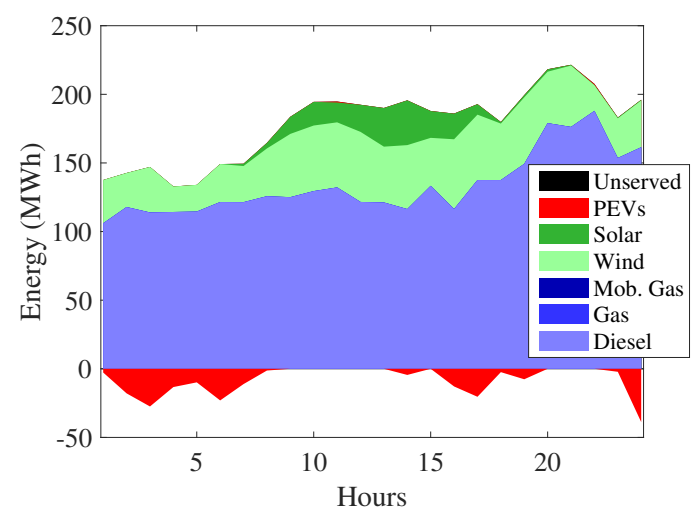

(a) Day-ahead energy market

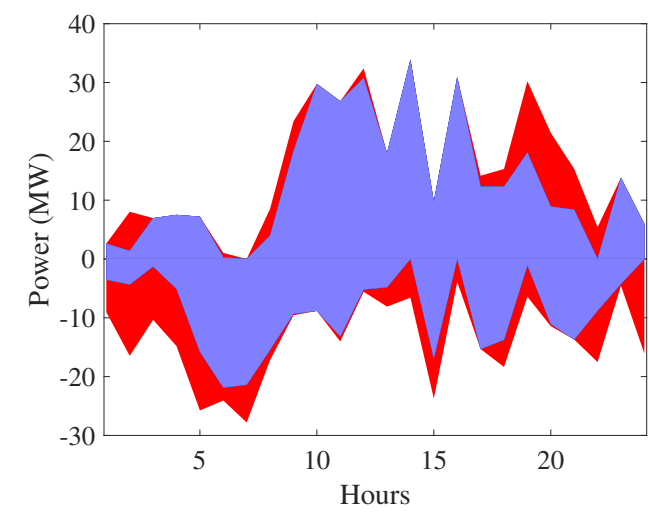

(b) Reserve capacity market

Figure 5: Day-ahead energy and reserve capacity for a single day

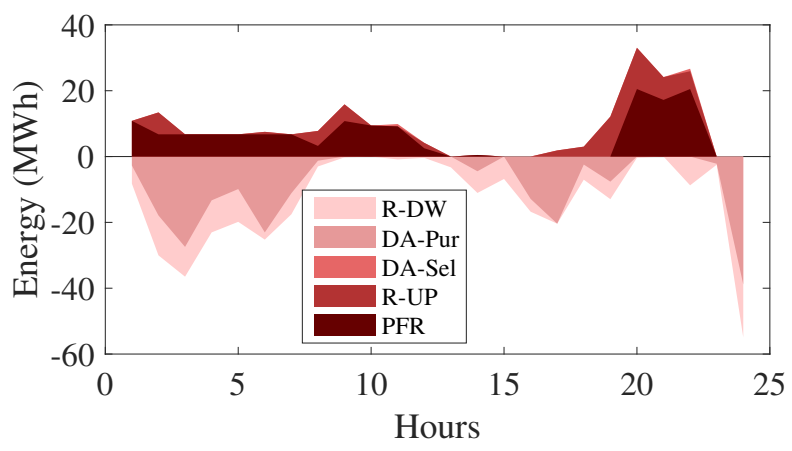

Figure 6: Day-ahead PEVs scheduling for a single day

The participation of electric vehicles in the different markets is represented in Figure 6. Positive values in this figure indicate the sales of energy in the day-ahead market (DA-Sel) and the participation in up-reserve capacity (R-UP) and PFR. On the other hand, the purchases in the day-ahead energy market (DA-Pur) and the participation in the down-reserve capacity market (R-DW) are represented using negative values. Observe that, for the considered day, most of the volume of the traded energy corresponds to purchases in the day-ahead energy market and PFR.

Figure 7 represents the quantity of power that the system has available for frequency primary response for different cases. For the sake of clarity, the capacity of the largest unit committed and the total expected cost (EC) in each case are also included. The available power of conventional units has been computed considering the capacity, the ramp limits and the droop of each unit. The contribution of each PEVs type in each bus and period for the frequency primary response is determined by variable $c_{k n t}^{\mathrm{V}, \mathrm{PR}}$. Figure 7 a represents the case in which PFR is not considered in the day-ahead market scheduling. Observe that in this case the available capacity of conventional power units is not enough to supply the production of the largest committed 
unit in the case of failure of this unit. Figures $7 \mathrm{~b}-7 \mathrm{~d}$ depict the available capacity for PFR considering different capacity costs, $C_{k n t}^{\mathrm{V}, \mathrm{PR}}$, offered by the PEVs. Observe in Figure $7 \mathrm{~b}$ that if $C_{k n t}^{\mathrm{V}, \mathrm{PR}}$ is equal to $€ 100 / \mathrm{MW}$, PEVs do not participate in PFR. However, as the capacity cost decreases, the participation of PEVs in the PFR increases while the total expected cost is reduced.

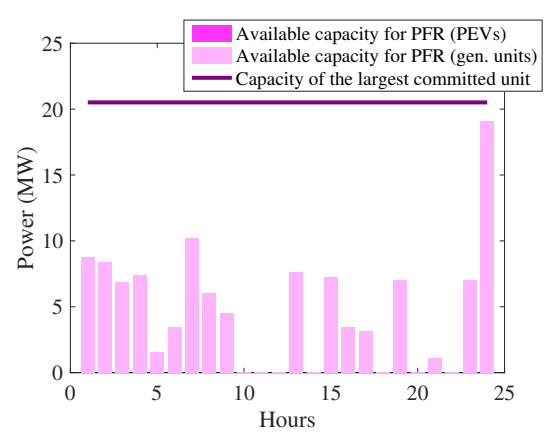

(a) $\mathrm{w} / \mathrm{o}$ PFR, EC $=434.4 \mathrm{k} €$

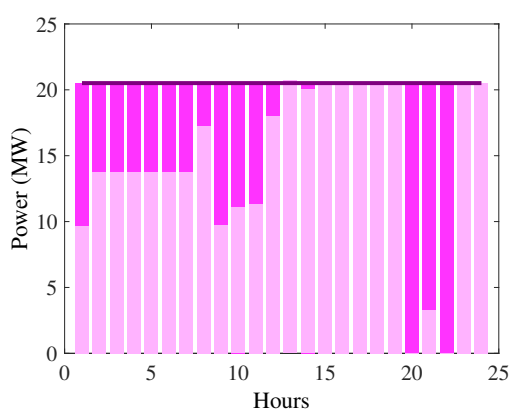

(c) $C_{k n t}^{\mathrm{V}, \mathrm{PR}}=€ 5 / \mathrm{MW}, \mathrm{EC}=435.9 \mathrm{k} €$

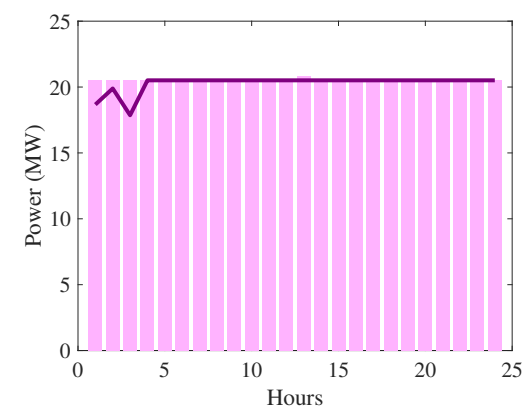

(b) $C_{k n t}^{\mathrm{V}, \mathrm{PR}}=€ 100 / \mathrm{MW}, \mathrm{EC}=438.1 \mathrm{k} €$

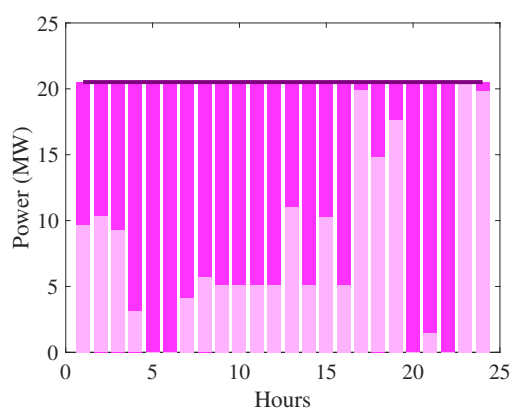

(d) $C_{k n t}^{\mathrm{V}, \mathrm{PR}}=€ 0.5 / \mathrm{MW}, \mathrm{EC}=435.1 \mathrm{k} €$

Figure 7: Available power for PFR

\section{Case 2). One-year simulation}

In order to test the performance of the formulation of problem (P1) in a longer period of time, the proposed day-ahead market scheduling procedure has been applied iteratively for each of the 365 days of 2015. For every single day, demand, wind and solar PV power availabilities are generated using sets of 12 scenarios as explained in Section 5.1. The daily driven distance for each electric vehicle is randomly generated using a normal distribution with mean and standard deviation equal to 35 and 9.6 , respectively, as done in [33]. The daily driven distance is multiplied by factor 0.7 in those days pertaining to weekends and holidays.

Considering the above, the following cases are solved:

- Base: Electric vehicles are allowed to participate in energy, reserve capacity and PFR services.

- NoF: Electric vehicles are not allowed to participate in PFR. 
- NoR: Electric vehicles are not allowed to participate in the reserve capacity market and PFR.

- NoD: Electric vehicles are not allowed to sell energy in the day-ahead energy market, and to participate in the reserve capacity market and PFR.

- Fixed: Electric vehicles charge their batteries uniformly during their charging period and they are not allowed to sell energy in the day-ahead energy market, and to participate in the reserve capacity market and in PFR.

Each of the daily simulations of the base case has been solved in a time smaller than 1 hour, with a mean solution time equal to 283 seconds. The mean and maximum numbers of needed iterations considering the 365 simulated days have been 2.1 and 12 , respectively.

The relevant results obtained are summarized in Table 5. This table lists the resulting annual expected operating costs and the number of startups of generating units for different values of renewable penetration. Note that the renewable penetration factor indicates the considered renewable capacity in each case study with respect to the values provided in Table 2 . In order to facilitate the comparison between the different cases, the values associated with Fixed case are expressed in absolute terms, whereas the values for the rest of cases are expressed in terms of percentage with respect to those obtained in the Fixed case.

Table 5: Sensitivity analysis results

\begin{tabular}{c|l|c|c|c|c|c}
\hline $\begin{array}{c}\text { Renewable } \\
\text { penetration factor }\end{array}$ & Magnitude & $\begin{array}{c}\text { Fixed } \\
(\text { abs. })\end{array}$ & $\begin{array}{c}\text { NoD } \\
(\%)\end{array}$ & $\begin{array}{c}\text { NoR } \\
(\%)\end{array}$ & $\begin{array}{c}\text { NoF } \\
(\%)\end{array}$ & $\begin{array}{c}\text { Base } \\
(\%)\end{array}$ \\
\hline \multirow{2}{*}{$\times 0.5$} & AC (M€) & 216.3 & 0.02 & -0.39 & -0.61 & -0.74 \\
& NS (\#) & 3573 & -3.39 & -14.39 & -18.14 & -25.16 \\
\hline \multirow{2}{*}{$\times 1$} & AC (M€) & 200.5 & -0.42 & -0.46 & -0.77 & -1.49 \\
& NS (\#) & 3688 & -2.87 & -18.17 & -22.75 & -48.67 \\
\hline \multirow{2}{*}{$\times 2$} & AC (M€) & 178.1 & -0.16 & -0.75 & -1.03 & -4.36 \\
& NS (\#) & 4673 & -5.03 & -29.49 & -27.93 & -47.72 \\
\hline
\end{tabular}

As expected, the Table 5 shows that the annual operating costs are highly reduced as the renewable penetration increases. In the same vain, the annual operating costs $(\mathrm{AC})$ decrease as PEVs are more involved in the operation of the power system. Besides, it can be observed that this reduction is higher as long as the renewable penetration increases. It is remarkable the $4.36 \%$ reduction of the cost attained by the Base case with respect to the Fixed case for a high renewable penetration factor.

Table 5 also provides a measure of the cycling of the conventional units in terms of the total number of startups during the year (NS). The cycling can be defined as the changing in the power output of conventional units by means of ramping and switchings. We observe that the 
cycling decreases appreciably with the participation of PEVs in the different trading floors. The decrease in the number of startups when PEVs participate in PFR is significant. For instance, in the case with installed renewable capacity factor equal to 1, the participation of PEVs in PFR reduces the total number of startups from 2408, in NoF case, to 1712 in Base case.

Case 3). Analysis of the economic performance of PEVs

In this case we analyze the economic results of the participation of PEVs in the different electricity markets described in Section 2.1 considering the pricing mechanism described in Section 3 and the degradation costs suffered by the usage of the batteries of PEVs.

For simplicity, the input data described in Case 1 are used in this case study. Table 6 provides the expected energy charged and discharged from each PEV group in the instances Fixed, NoD, NoR, NoF and Base defined in Case 2. Observe that the smallest quantity of energy charged is given in Fixed case. Note that in this case it is enforced that the amount of energy needed for the daily usage of PEVs is uniformly charged during the charging interval of each PEV group, whereas the amounts of energy charged in the rest of cases result from clearing the day-ahead scheduling. Therefore, the final amount of energy charged by each PEV group depends on the offering strategies of PEVs and the rest of agents participating in the different trading floors. It is also observed in Table 6 that the amount of energy discharged is significantly less than the energy charged for most of the PEV groups and cases analyzed. Only in case $N o R$, in which PEVs are not allowed to participate in reserve capacity and PFR markets, it is observed a comparatively higher amount of energy discharged in PEVs belonging to group 3. It is also observed that the expected energy charged and discharged decreases if PEVs are allowed to participate in capacity markets (NoF and Base) cases. This is so because the participation in capacity markets does not always entail the physical deployment of energy.

Table 6: Expected charged and discharged energy (MWh)

\begin{tabular}{c|cc|cc|cc|cc|cr}
\hline \multirow{2}{*}{ Group } & \multicolumn{10}{c}{ Case } \\
\cline { 2 - 11 } & \multicolumn{2}{|c}{ Fixed } & \multicolumn{1}{c}{ NoD } & \multicolumn{1}{c}{ NoR } & \multicolumn{2}{c}{ NoF } & Base \\
\cline { 2 - 11 } & EC & ED & EC & ED & EC & ED & EC & ED & EC & ED \\
\hline 1 & 26.6 & 0.0 & 83.2 & 0.0 & 82.2 & 0.0 & 86.5 & 3.2 & 84.7 & 2.9 \\
2 & 18.5 & 0.0 & 58.2 & 0.0 & 63.0 & 6.3 & 68.6 & 9.5 & 59.7 & 2.8 \\
3 & 8.0 & 0.0 & 18.9 & 0.0 & 37.8 & 18.3 & 24.8 & 6.2 & 19.9 & 1.7 \\
\hline Total & 53.1 & 0.0 & 160.3 & 0.0 & 183.0 & 24.6 & 179.9 & 18.9 & 164.3 & 7.4 \\
\hline
\end{tabular}

Table 7 provides the expected costs incurred by PEVs in the different trading floors considering the pricing mechanism described in Section 3. Due to space limitations, aggregated data considering all PEVs are provided. Note that negative values in this table represent expected revenues. In this manner, Table 7 shows that most of the expected costs faced by PEVs result 
from purchasing energy in the day-ahead energy market, whereas the participation in the rest of markets has associated significantly smaller revenues and costs for PEVs. As expected, the lowest expected cost is obtained in the Fixed case in which, as observed in Table 6, a comparatively small quantity of energy is charged in PEVs.

Table 7: Expected cost $(\mathrm{k} €)$

\begin{tabular}{l|c|c|c|c|c}
\hline Market & Fixed & NoD & NoR & NoF & Base \\
\hline DA EP & 11.8 & 34.1 & 34.5 & 33.1 & 29.3 \\
DA ES & 0.0 & 0.0 & -3.9 & -2.5 & -0.3 \\
DA U-RC & 0.0 & 0.0 & 0.0 & -0.8 & -0.5 \\
DA D-RC & 0.0 & 0.0 & 0.0 & -0.8 & -0.8 \\
PFR & 0.0 & 0.0 & 0.0 & 0.0 & -0.6 \\
RT U-RD & 0.0 & 0.0 & 0.0 & -0.9 & -0.2 \\
RT D-RD & 0.0 & 0.0 & 0.0 & 0.7 & 0.5 \\
\hline Total & 11.8 & 34.1 & 30.6 & 28.9 & 27.4 \\
\hline
\end{tabular}

DA U-RC: Day-ahead up reserve capacity; DA D-RC: Day-ahead down reserve capacity

RT U-RD: Real-time up reserve deployment; RT D-RD: Real-time down reserve deployment

In order to compute properly the costs incurred by PEVs, battery degradation costs are explicitly considered in this case study. In this manner, the costs of battery degradation are calculated using the linear regression model described in [48], which models the battery degradation in PEVs with vehicle-to-grid capability assuming that the capacity degradation of the batteries is a linear function of the number of round trip cycles. Considering that batteries are retired when their capacity retention is less than $80 \%$ of their initial capacity and the battery performance analyzed in [49], a battery life of $N^{\mathrm{Cyc}}=4370$ round trip cycles has been assumed. In this manner, for a given battery cost, $C^{\text {Bat }}(€ / \mathrm{MWh})$, the expected battery degradation cost of PEV group $k$ in bus $n$ is estimated as follows:

$$
C_{n k}^{\mathrm{Deg}}=\frac{C^{\mathrm{Bat}}}{N^{\mathrm{Cyc}}} \sum_{t \in T_{k}} \sum_{\omega \in \Omega} \sum_{c \in C} \pi_{\omega} \tau_{c} e_{k n t \omega c}^{\mathrm{C}}, \quad \forall n \in N, \forall k \in K .
$$

where $\pi_{w}$ is the probability of scenario $\omega, \tau_{c}$ is the probability of contingency $c$ and $e_{k n t \omega c}^{\mathrm{C}}$ is the energy charged by PEV group $k$, in bus $n$, period $t$, scenario $\omega$ and contingency $c$.

Table 8 provides the expected battery degradation cost and the total costs per net charged energy incurred by each group of PEVs. The total expected cost per net charged energy is computed as the sum of the expected degradation cost plus the sum of the expected costs incurred by PEVs in the different trading floors, divided by the net amount of energy charged (expected energy charged minus expected energy discharged) in the batteries of PEVs. This value is used to compare the economic performance of PEVs considering cases with different amounts of energy charged. Two different battery costs, equal to 200 and $100 € / \mathrm{kWh}$, are 
considered. According to [50], the current cost of the batteries installed in new PEV models is $€ 200 / \mathrm{kWh}$, whereas $€ 100 / \mathrm{kWh}$ is the estimate of the battery cost expected to be reached before the end of 2022, as claimed by relevant automobile manufacture companies as General Motors Company or Tesla.

Table 8: Expected degradation cost and expected cost per MWh

\begin{tabular}{|c|c|c|c|c|c|c|c|c|c|c|c|}
\hline \multirow{3}{*}{$\begin{array}{c}\text { B. cost } \\
(€ / \mathrm{kWh})\end{array}$} & \multirow{3}{*}{ Group } & \multicolumn{10}{|c|}{ Case } \\
\hline & & \multicolumn{2}{|c|}{ Fixed } & \multicolumn{2}{|c|}{ NoD } & \multicolumn{2}{|c|}{ NoR } & \multicolumn{2}{|c|}{ NoF } & \multicolumn{2}{|c|}{ Base } \\
\hline & & $\overline{E D C}$ & ECE & EDC & ECE & EDC & ECE & EDC & ECE & EDC & ECE \\
\hline \multirow{4}{*}{200} & 1 & 1.2 & 254.3 & 3.8 & 262.9 & 3.8 & 259.0 & 4.0 & 239.9 & 3.8 & 226.6 \\
\hline & 2 & 0.9 & 264.4 & 2.6 & 262.2 & 2.9 & 267.8 & 3.1 & 238.4 & 2.8 & 243.7 \\
\hline & 3 & 0.4 & 324.7 & 0.9 & 227.8 & 1.7 & 125.9 & 1.1 & 160.1 & 0.9 & 139.5 \\
\hline & Total & 2.5 & 268.4 & 7.3 & 257.4 & 8.4 & 242.1 & 8.2 & 227.4 & 7.5 & 219.5 \\
\hline \multirow{4}{*}{100} & 1 & 0.6 & 231.4 & 1.9 & 240.0 & 1.9 & 236.1 & 2.0 & 216.2 & 1.9 & 202.9 \\
\hline & 2 & 0.4 & 241.6 & 1.3 & 239.3 & 1.4 & 242.4 & 1.6 & 211.9 & 1.3 & 219.6 \\
\hline & 3 & 0.3 & 301.8 & 0.4 & 204.9 & 0.8 & 81.3 & 0.6 & 129.7 & 0.4 & 114.4 \\
\hline & Total & 1.3 & 245.5 & 3.6 & 234.5 & 4.1 & 215.1 & 4.2 & 201.7 & 3.6 & 195.5 \\
\hline
\end{tabular}

In Table 8 it is observed that, for the considered day, the lowest expected battery degradation costs are obtained in Fixed case. This result is coherent with the usage of the battery provided in Table 6. Additionally, the results provided in Table 8 show that the Base case, in which PEVs are allowed to participate in all considered trading floors, attains the smallest expected cost per net charged energy. Especially, it is observed a high decrement in the expected cost if PEVs are allowed to participate in reserve and PFR capacity markets. This result is even more acute if a low battery cost $(100 € / \mathrm{kWh})$ is considered.

Finally, it is analyzed the effect of the reduction of the lifetime of the batteries caused by the vehicle-to-grid operation. Table 9 provides the expected cost per net charged energy obtained in Base case considering different values modeling the reduction of the estimated lifetime of the batteries. In this manner, several cases considering a lifetime of the battery ranging between 100 and $50 \%$ of the nominal number of round trip cycles, 4370, have been simulated. As expected, a reduction in the lifetime of the battery causes an increment in the expected cost per net energy charged in the batteries of PEVs. As an example, the expected cost per energy charged considering all PEVs increases 20.9 and $12.3 \%$ for battery costs equal to 200 and 100 $€ / \mathrm{kWh}$, respectively, when the lifetime of the battery is reduced by half. However, note that these expected costs outperform those obtained for Fixed and $N o D$ cases shown in Table 8, in which the lifetime of the battery is considered to be equal to its nominal value corresponding with 4370 round trip cycles. 
Table 9: Expected cost per MWh for different battery lifetimes in the Base case (€/MWh)

\begin{tabular}{c|c|cccccc}
\hline $\begin{array}{c}\text { B. cost } \\
(€ / \mathrm{kWh})\end{array}$ & \multirow{2}{*}{ Group } & \multicolumn{5}{|c}{ Lifetime of the battery } & over the nominal value \\
\cline { 3 - 8 } & $100 \%$ & $90 \%$ & $80 \%$ & $70 \%$ & $60 \%$ & $50 \%$ \\
\hline \multirow{3}{*}{200} & 1 & 226.6 & 231.9 & 238.5 & 247.0 & 258.3 & 274.1 \\
& 2 & 243.7 & 249.0 & 255.6 & 264.2 & 275.7 & 285.5 \\
& 3 & 139.5 & 145.0 & 152.0 & 160.9 & 172.8 & 189.4 \\
\cline { 2 - 8 } & Total & 219.5 & 224.8 & 231.5 & 240.1 & 251.5 & 265.4 \\
\hline \multirow{3}{*}{100} & 1 & 202.9 & 205.5 & 208.8 & 213.1 & 218.7 & 226.6 \\
& 2 & 219.6 & 222.3 & 225.6 & 229.9 & 235.7 & 243.7 \\
& 3 & 114.4 & 117.3 & 120.7 & 125.2 & 131.1 & 139.5 \\
\cline { 2 - 8 } & Total & 195.5 & 198.2 & 201.5 & 205.8 & 211.5 & 219.5 \\
\hline
\end{tabular}

\section{Summary and conclusions}

In this paper, we have formulated a day-ahead scheduling for isolated power systems with high penetration of renewable power units and electric vehicles considering day-ahead energy and reserve capacity markets, as well as primary frequency response. The uncertainty of demand and renewable power availability has been explicitly considered using a stochastic programming approach. An N-1 security criterion has been applied in order to compute the primary frequency response needs of the system. The resulting problem has been formulated as a mixed-integer programming problem and an iterative solution procedure has been proposed. A procedure for settling the financial remuneration of the electricity market participants has also been derived.

The proposed formulation has been applied to a real isolated power system located in Spain. The numerical results show that the operating cost of the system can be significantly reduced if PEVs participate in the different markets. PEVs are actively involved in the purchase of energy in the day-ahead energy market and in the participation in the down-reserve capacity and PFRs markets. Moreover, we have observed that the cycling of conventional units can be drastically reduced thanks to the incorporation of PEVs in the reserve capacity and the PFR markets. It has been also verified that the contribution of PEVs to the power system operation is more beneficial in terms of cost reduction as the penetration of renewable energies increases. Finally, the obtained results indicate that the active participation of PEVs in the different electricity markets may be profitable for PEVs from an economical point of view.

\section{Acknowledgment}

This work has been supported by the Ministry of Economy and Competitiveness of Spain under Project DPI2015-71280-R MINECO/FEDER, UE. 


\section{References}

\section{References}

[1] The Paris Agreement. Available from: http://unfccc.int/paris_agreement/items/948 5.php

[2] International Renewable Energy Agency (IRENA) (2016). Renewable energy statistics 2016. Available from: http://www.irena.org

[3] Spanish Network Operator. Available from: http://www.ree.es.

[4] Margaris I, Hansen A, Cutululis N, Sørensen P, Hatziargyriou N. Impact of wind power in autonomous power systems - power fluctuations-modelling and control issues, Wind Energy 2011; 14(1):133-53.

[5] Dunn B, Kamath H, Tarascon JM. Electrical Energy Storage for the Grid: A Battery of Choices. Science 2011; 334:928-35.

[6] Tuttle DP, Baldick R. The evolution of plug-in electric vehicle-grid interactions. Operat Res 2011;3(1):500-5.

[7] Brooks A. Vehicle-to-grid demonstration project: Grid regulation ancillary service with a battery electric vehicle. AC Propulsion, Inc. 2002; Sponsored by California Air Resources Board Contract $01-313$.

[8] Kempton $\mathrm{W}$ et al. A test of vehicle-to-grid (V2G) for energy storage and frequency regulation in the PJM system. Dept. Elect. Comput. Eng., Univ. Delaware, Newark, DE, USA. Available from: http:// www.udel.edu/V2G/resources/test-v2g-in-pjm-jan09.pdf.

[9] Uddin K, Dubarry M, Glick, MB. The viability of vehicle-to-grid operations from a battery technology and policy perspective. Energy Policy 2018:113;342-7.

[10] Asensio M, Contreras J. Stochastic Unit Commitment in Isolated Systems With Renewable Penetration Under CVaR Assessment. IEEE Transactions on Smart Grids 2016:7(3);13561367.

[11] Psarros GN, Nanou SI, Papaefthymiou SV, Papathanassiou SA. Generation Scheduling in Non-Interconnected Islands with high RES penetration. Renewable Energy 2017:1—20.

[12] Papathanassiou SA, Boulaxis NG. Power limitations and energy yield evaluation for wind farms operating in island systems. Renewable Energy 2006:31(4);457--79.

[13] Chang CA, Wu YK, Chen BK. Determination of maximum wind power penetration in an isolated island system by considering spinning reserve. Energies 2006:9. 
[14] Thomas D, Deblecker O, Ioakimidis CS. Optimal design and techno-economic analysis of an autonomous small isolated microgrid aiming at high RES penetration. Energy 2016:116;364--79.

[15] Pascal M, Rachid C, Alexandre O. Optimizing a battery energy storage system for frequency control application in an isolated power system. IEEE Trans Power Syst 2009:24(3);1469--77.

[16] Munoz ER, Razeghi G, Zhang L et al. Electric vehicle charging algorithms for coordination of the grid and distribution transformer levels. Energy 2016:113;930-42.

[17] Wang L, Sharkh S, Chipperfield A. Optimal coordination of vehicle-to-grid batteries and renewable generators in a distribution system. Energy 2016:113;1250-64.

[18] Huang SJ, Yang J, Li SJ. Black-Scholes option pricing strategy and risk-averse coordination for designing vehicle-to-grid reserve contracts. Energy 2017:137;325-35.

[19] Bellekom S, Benders R, Pelgröm S, Moll H. Electric cars and wind energy: Two problems, one solution? A study to combine wind energy and electric cars in 2020 in The Netherlands. Energy 2012:45(1);859-66.

[20] Graabak I, Wu Q, Warland L, Liu Z. Optimal planning of the Nordic transmission system with $100 \%$ electric vehicle penetration of passenger cars by 2050. Energy 2016:107;648 — 60 .

[21] Ciechanowicz D, Knoll A, Osswald P, Pelzer D. Towards a Business Case for Vehicle-toGrid-Maximizing Profits in Ancillary Service Markets. In: Rajakaruna S., Shahnia F., Ghosh A. (eds) Plug In Electric Vehicles in Smart Grids. Power Systems. (2015) Springer, Singapore.

[22] Binding C, Gantenbein D, Jansen B, Sundström O, Andersen, P B, Marra F, Træholt C. Electric vehicle fleet integration in the Danish EDISON project - A virtual power plant on the island of Bornholm. In IEEE PES General Meeting, PES 2010.

[23] Lopes JAP, Almeida PMR, Soares FJ. Using vehicle-to-grid to maximize the integration of intermittent renewable energy resources in islanded electric grids. 2009 International Conference on Clean Electrical Power, ICCEP:290 - 95.

[24] Almeida PMR, Lopes JAP, Soares FJ, Seca L. Electric vehicles participating in frequency control: Operating islanded systems with large penetration of renewable power sources. 2011 IEEE PES Trondheim PowerTech: The Power of Technology for a Sustainable Society, POWERTECH 2011:1;5--10. 
[25] Mu Y, Wu J, Ekanayake J, Jenkins N, Jia H. Primary frequency response from electric vehicles in the Great Britain power system. IEEE Transactions on Smart Grid 2013:4(2);114250.

[26] Donadee J, Ilic MD. Stochastic optimization of grid to vehicle frequency regulation capacity bids. IEEE Trans. Smart Grid 2014:5(2);1061—69.

[27] Liu H, Hu ZC, Song YH, Lin J. Decentralized vehicle-to-grid control for primary frequency regulation considering charging demands. IEEE Trans. Power syst 2013:28(3);3480-3489

[28] Liu H, Hu Z, Song Y, Wang J, Xie X. Vehicle-to-Grid Control for Supplementary Frequency Regulation Considering Charging Demands. IEEE Transactions on Power Systems 2015:30(6);3110-19.

[29] Vagropoulos SI, Kyriazidis DK, Bakirtzis AG. Real-time charging management framework for electric vehicle aggregators in a market environment. IEEE Trans. Smart Grid 2016:7(2);948-57.

[30] Yao E, Wong VWS, Schober R. Robust Frequency Regulation Capacity Scheduling Algorithm for Electric Vehicles. IEEE Transactions on Smart Grids 2017:8(2); 984—97.

[31] Sun, S, Dong, M, Liang, B. Real-Time Welfare-Maximizing Regulation Allocation in Dynamic Aggregator-EVs System. IEEE Transactions on Smart Grids 2013:5(3); 1397-1409.

[32] Morales JM, Conejo AJ, Liu K, Zhong J. Pricing electricity in pools with wind producers. IEEE Trans Power Syst 2012;27(3):1366-76.

[33] Carrión M, Zárate-Miñano R. Operation of intermittent-dominated power systems with a significant penetration of plug-in electric vehicles. Energy 2015:90;827-35.

[34] Gómez-Expósito A, Conejo AJ, Cañizares C. Electric energy systems: analysis and operation. Boca Raton: CRC Press 2008.

[35] Restrepo J F, Galiana FD. Unit commitment with primary frequency regulation constraints. IEE Transactions on Power Systems 2005:20(4);1836--42.

[36] Vaya MG, Andersson G. Optimal bidding strategy of a plug-in electric vehicle aggregator in day-ahead electricity markets. IEEE Transactions on Power Systems 2013:24(4);1855-66 
[37] Sarker MR, Dvorkin Y, Ortega-Vazquez MA. Optimal participation of an electric vehicle aggregator in day-ahead energy and reserve markets. IEEE Transactions on Power Systems 2016:31(5);3506-15.

[38] Carrión M, Arroyo JM. A Computationally Efficient Mixed-Integer Linear Formulation for the Thermal Unit Commitment Problem. IEEE Transactions on Power Systems 2006:21(3);1371-78.

[39] Ardakani AJ, Bouffard F. Identification of Umbrella Constraints in DC-Based SecurityConstrained Optimal Power Flow IEEE Transactions on Power Systems 2013:28(4);392434.

[40] Canary Islands Power System. Available from: http://www.ree.es/es/actividades/sistemaelectrico-canario/red-de-transporte

[41] Government of the Canary Islands, Energetic plan of the Canary Islands, PECAN 2007. Available from: http://www.gobcan.es/ceic/energia/doc/planificacion/pecan/pecan2002.pdf (Spanish)

[42] Mobility report for residents in Spain (MOVILIA). Available from: http://www.fomento.es.

[43] Specifications of the Renault Zoe. Available from: https://www.renault.co.uk/vehicles/newvehicles/zoe-250.html

[44] National Meteorological Agency of Spain. Available from: http://www.aemet.es.

[45] Danish Wind Industry Association, Wind Turbine Power Calculator. Available from: http://www.windpower.org.

[46] Conejo AJ, Carrión M, Morales JM. Decision Making Under Uncertainty in Electricity Markets. New York, NY: Springer; 2010.

[47] Gröwe-Kuska N, Heitsch H, Römisch W. Scenario reduction and scenario tree construction for power management problems.Proc. IEEE Bologna Power Technology Conf. Bologna, Italy; 2003.

[48] Ortega-Vazquez MA, Optimal scheduling of electric vehicle charging and vehicle-to-grid services at household level including battery degradation and price uncertainty. IET Generation, Transmission \& Distribution, 2014:8(6);1007--1016. 
[49] Stroe D, Swierczynski M. Accelerated aging of lithium-ion batteries based on electric vehicle mission profile. IEEE Energy Conversion Congress and Exposition (ECCE), Cincinnati, OH, 2017; 5631-5637.

[50] International Energy Agency. Global EV Outlook 2017. Available from https://www.iea.org/publications/freepublications/publication/

GlobalEVOutlook2017.pdf 


\section{Appendix A. Notation}

The notation used throughout the paper is included below for quick reference.

Indices

$c$ Index of contingencies

$F(\ell)$ Destination or receiving bus of line $\ell$

$g$ Index of generating units

$k$ Index of plug-in electric vehicle (PEV) groups

$\ell$ Index of transmission lines

$n$ Index of buses

$O(\ell)$ Origin or sending bus of line $\ell$

$t$ Index of time periods

$\omega$ Index of scenarios

Sets

$C$ Set of post-contingency states

$G$ Set of generating units

$G_{n}$ Set of generating units located in bus $n$

$G^{\mathrm{C}}$ Set of dispatchable generating units

$G_{n}^{\mathrm{C}}$ Set of dispatchable generating units located in bus $n$

$G^{\mathrm{I}}$ Set of intermittent generating units

$G_{n}^{\mathrm{I}}$ Set of intermittent generating units located in bus $n$

$K$ Set of PEV groups

$K_{t}$ Set of PEV groups that are connected to the grid in period $t$

$L$ Set of transmission lines

$L_{n}^{\mathrm{F}}$ Set of transmission lines whose destination bus is $n$

$L_{n}^{\mathrm{O}}$ Set of transmission lines whose origin bus is $n$

$N$ Set of buses

$T$ Set of time periods

$T_{k}$ Set of time periods in which PEV group $k$ can be charged from or discharged to the grid

$\Omega$ Set of scenarios 


\section{Variables}

$c_{n k}^{\text {Deg }}$ Expected battery degradation cost of PEVs belonging to group $k$ in bus $n$.

$c_{g t}^{\mathrm{dw}}$ Scheduled down reserve capacity by unit $g$ in period $t$

$c_{g t}^{\text {up }}$ Scheduled up reserve capacity by unit $g$ in period $t$

$c_{k n t}^{\mathrm{V}, \mathrm{dw}}$ Scheduled down reserve capacity by PEV group $k$ in bus $n$ and period $t$

$c_{k n t}^{\mathrm{V} \text {,up }}$ Scheduled up reserve capacity by PEV group $k$ in bus $n$ and period $t$

$c_{k n t}^{\mathrm{V}, \mathrm{PR}}$ Scheduled capacity that can be used for primary frequency response by PEV group $k$ in bus $n$ and period $t$

$s_{g t}^{\text {sh }}$ Shutdown cost of unit $g$ in period $t$

$s_{g t}^{\text {st }}$ Startup cost of unit $g$ in period $t$

$e_{k n t \omega c}^{\mathrm{D}}$ Energy discharged by PEV group $k$ in bus $n$, period $t$, scenario $\omega$ and post-contingency state $c$

$e_{k n t \omega c}^{\mathrm{C}}$ Energy charged by PEV group $k$ in bus $n$, period $t$, scenario $\omega$ and post-contingency state $c$

$p_{g t}^{\mathrm{DA}}$ Power scheduled in the day-ahead market by unit $g$ in period $t$

$p_{k n t}^{\mathrm{V}, \mathrm{DAC}}$ Charge power scheduled in the day-ahead market by PEV group $k$ in bus $n$ and period $t$

$p_{k n t}^{\mathrm{V}, \mathrm{DAD}}$ Discharge power scheduled in the day-ahead market by PEV group $k$ in bus $n$ and period $t$

$p_{k n t \omega c}^{\mathrm{V}, \mathrm{PR}}$ Primary response output of PEV group $k$ in bus $n$, period $t$ and scenario $\omega$ and postcontingency state $c$.

$p_{k n t \omega c}^{\mathrm{V}, \mathrm{PRC}}$ Primary response output provided by PEV group $k$ in charging mode in bus $n$, period $t$ and scenario $\omega$ and post-contingency state $c$.

$p_{k n t \omega c}^{\mathrm{V}, \mathrm{PRD}}$ Primary response output provided by PEV group $k$ in discharging mode in bus $n$, period $t$ and scenario $\omega$ and post-contingency state $c$.

$p_{\ell t}^{\mathrm{L}, \mathrm{DA}}$ Power flow resulting from the day-ahead schedule in line $\ell$ and period $t$

$p_{\ell t \omega}^{\mathrm{L}, \mathrm{RT}}$ Power flow resulting from the real-time dispatch in line $\ell$ in period $t$ and scenario $\omega$

$p_{g t \omega c}^{\mathrm{PR}}$ Primary response output of unit $g$ in period $t$ and scenario $\omega$ and post-contingency state $c$

$p_{g t \omega}^{\mathrm{RT}}$ Power generated in the real-time by unit $g$ in period $t$ and scenario $\omega$

$p_{n t \omega}^{\mathrm{U}}$ Unserved demand in bus $n$, period $t$ and scenario $\omega$

$p_{n t \omega c}^{\mathrm{U}, \mathrm{PR}}$ Unserved demand in bus $n$, period $t$, scenario $\omega$ and post-contingency state $c$

$r_{g t \omega}^{\mathrm{dw}}$ Deployed down reserve in the real-time dispatch by unit $g$ in period $t$ and scenario $\omega$

$r_{g t \omega}^{\mathrm{up}}$ Deployed up reserve in the real-time dispatch by unit $g$ in period $t$ and scenario $\omega$

$r_{k n t \omega}^{\mathrm{V}, \mathrm{dw}}$ Deployed down reserve in the real-time dispatch by PEV group $k$ in bus $n$, period $t$ and scenario $\omega$

$r_{k n t \omega}^{\mathrm{V}, \mathrm{dwC}}$ Deployed down reserve in the real-time dispatch by PEV group $k$ in charging mode in bus $n$, period $t$ and scenario $\omega$

$r_{k n t \omega}^{\mathrm{V}, \mathrm{dwD}}$ Deployed down reserve in the real-time dispatch by PEV group $k$ in discharging mode in bus $n$, period $t$ and scenario $\omega$

$r_{k n t \omega}^{\mathrm{V} \text {,up }}$ Deployed up reserve in the real-time dispatch by PEV group $k$ in bus $n$, period $t$ and scenario $\omega$

$r_{k n t \omega}^{\mathrm{V} \text {,upC }}$ Deployed up reserve in the real-time dispatch by PEV group $k$ in charging mode in bus $n$, period $t$ and scenario $\omega$ 
$r_{k n t \omega}^{\mathrm{V}, \text { upD }}$ Deployed up scheduled reserve in the real-time dispatch by PEV group $k$ in discharging mode in bus $n$, period $t$ and scenario $\omega$

$p_{g t}^{\mathrm{S}, \mathrm{DA}}$ Power spillage of intermittent unit $g$ in period $t$ in the day-ahead market

$p_{g t \omega}^{\mathrm{S}, \mathrm{RT}}$ Power spillage of intermittent unit $g$ in period $t$ and scenario $\omega$ in the real-time dispatch

$\operatorname{soc}_{k n t \omega c}$ Energy stored by PEV group $k$ in bus $n$, period $t$, scenario $\omega$ and post-contingency state $c$

$v_{g t}$ Binary variable that is equal to 1 if dispatchable unit $g$ is on line in period $t$, being equal to 0 otherwise

$\Delta f_{t \omega c}$ System frequency variation in period $t$, scenario $\omega$ and post-contingency state $c$

$\lambda_{n t}^{\mathrm{DA}}$ Dual variable used to compute the day-ahead energy price in bus $n$ and period $t$

$\lambda_{n t \omega}^{\mathrm{RT}}$ Dual variable used to compute the real-time energy price in bus $n$, period $t$ and scenario $\omega$

$\theta_{n t}^{\mathrm{DA}}$ Voltage bus angle resulting from the day-ahead schedule in bus $n$ and period $t$

$\theta_{n t \omega}^{\mathrm{RT}}$ Voltage bus angle resulting from the real-time dispatch in bus $n$, period $t$ and scenario $\omega$

Parameters

$C^{\text {Bat }}$ Cost of the battery of PEVs in (€/MWh)

$C_{g t}^{\mathrm{cdw}}$ Down reserve capacity cost offer of unit $g$ and period $t$

$C_{g t}^{\text {cup }} \mathrm{Up}$ reserve capacity cost offer of unit $g$ and period $t$

$C_{g t}^{\mathrm{DA}}$ Production cost offer of unit $g$ in the day-ahead market and period $t$

$C_{g t}^{\mathrm{rdw}}$ Deployed down reserve cost offer of unit $g$ and period $t$

$C_{g t}^{\text {rup }}$ Deployed up reserve cost offer of unit $g$ and period $t$

$C_{k n t}^{\mathrm{V} \text {,cdw }}$ Scheduled down reserve capacity cost offer of PEV group $k$ in bus $n$ and period $t$

$C_{k n t}^{\mathrm{V} \text {,cup }}$ Scheduled up reserve capacity cost offer of PEV group $k$ in bus $n$ and period $t$

$C_{k n t}^{\mathrm{V}, \mathrm{DAC}}$ Day-ahead charging cost offer of PEV group $k$ in bus $n$ and period $t$

$C_{k n t}^{\mathrm{V}, \mathrm{DAD}}$ Day-ahead discharging cost offer of PEV group $k$ in bus $n$ and period $t$

$C_{k n t}^{\mathrm{V} \text {,rdw }}$ Deployed down reserve cost offer of PEV group $k$ in bus $n$ and period $t$

$C_{k n t}^{\mathrm{V} \text {,rup }}$ Deployed up reserve cost offer of PEV group $k$ in bus $n$ and period $t$

$C_{k n t}^{\mathrm{V}, \mathrm{PR}}$ Primary frequency regulation capacity cost offer of PEV group $k$ in bus $n$ and period $t$

$C_{g}^{\text {sh }}$ Shutdown cost parameter of unit $g$

$C_{g}^{\text {st }}$ Startup cost parameter of unit $g$

$C^{\mathrm{U}}$ Cost of unserved demand

$D^{\mathrm{PR}}$ Maximum duration of the primary frequency response in hours

$L_{n t}^{\mathrm{DA}}$ Power demand in the day-ahead market in bus $n$ and period $t$

$L_{n t \omega}^{\mathrm{RT}}$ Power demand in the real-time dispatch in bus $n$, period $t$ and scenario $\omega$

$N^{\text {Cyc }}$ Number of complete charge/discharge cycles that the battery of a PEV can perform over its lifetime.

$N_{k n}^{\mathrm{V}}$ Number of PEVs in group $k$ and bus $n$

$P_{\max }^{\mathrm{V}}$ Maximum power charging/discharging rate of PEVs

$P_{\mathrm{dw}, g}^{\mathrm{G}}$ Ramp-down limit of unit $g$

$P_{\max , g}^{\mathrm{G}}$ Capacity of unit $g$ 
$P_{\text {min, },}^{\mathrm{G}}$ Minimum power output of unit $g$

$P_{\text {sh, } g}^{\mathrm{G}}$ Shutdown ramp limit of unit $g$

$P_{\mathrm{st}, g}^{\mathrm{G}}$ Startup ramp limit of unit $g$

$P_{\mathrm{up}, g}^{\mathrm{G}}$ Ramp-up limit of unit $g$

$P_{\max , \ell}^{\mathrm{L}}$ Capacity of line $\ell$

$R_{g}$ Frequency droop of unit $g$

$R^{\mathrm{V}}$ Frequency droop of PEVs

$S O C_{k n}^{0}$ Initial status of the batteries of PEVs in group $k$ and period $t$ at the beginning of the charging period

$S O C_{k n}^{\mathrm{F}}$ Minimum status of the batteries of PEVs in group $k$ and period $t$ at the end of the charging period

$S O C_{\max , k}$ Capacity of the batteries of PEVs in group $k$

$S O C_{\min , k}$ Minimum value of the energy that must remain in the batteries of PEVs in group $k$ in each charging/discharging period

$t_{k}^{\mathrm{F}}$ Final period in which PEV group $k$ can be charged or discharged

$t_{k}^{0}$ Initial period in which PEV group $k$ can be charged or discharged

$U_{g t}^{\mathrm{DA}}$ Availability of non-dispatchable unit $g$ in the day-ahead market and period $t$

$U_{g t \omega}^{\mathrm{RT}}$ Availability of non-dispatchable unit $g$ in the real-time dispatch in period $t$ and scenario $\omega$

$V_{g c}^{\text {post }}$ Binary parameter that is equal to 0 if outage of unit $g$ ocurrs in contingency $c$, and 1 otherwise.

$X_{\ell}$ Reactance of line $\ell$

$\alpha$ Efficiency of the charging/discharging processes of PEVs

$\Delta f_{\max }$ Maximum allowed frequency deviation

$\pi_{\omega}$ Probability of scenario $\omega$

$\tau_{c}$ Probability of post-contingency state $c$

\section{Appendix B. Complete formulation of the proposed model}

The detailed mathematical formulation of the two-stage stochastic programming problem denoted as (P1) is the following:

$$
\begin{aligned}
& \sum_{t \in T} \sum_{g \in G}\left(s_{g t}^{\mathrm{st}}+s_{g t}^{\mathrm{sh}}+C_{g t}^{\mathrm{DA}} p_{g t}^{\mathrm{DA}}+C_{g t}^{\mathrm{cup}} c_{g t}^{\mathrm{up}}+C_{g t}^{\mathrm{cdw}} c_{g t}^{\mathrm{dw}}\right)+ \\
& \sum_{t \in T} \sum_{n \in N} \sum_{k \in K_{t}}\left(C_{k n t}^{\mathrm{V}, \mathrm{DAD}} p_{k n t}^{\mathrm{V}, \mathrm{DAD}}-C_{k n t}^{\mathrm{V}, \mathrm{DAC}} p_{k n t}^{\mathrm{V}, \mathrm{DAC}}+\right. \\
& \left.C_{k n t}^{\mathrm{V}, \mathrm{cup}} c_{k n t}^{\mathrm{V}, \mathrm{up}}+C_{k n t}^{\mathrm{V}, \mathrm{cdw}} c_{k n t}^{\mathrm{V}, \mathrm{dw}}+C_{k n t}^{\mathrm{V}, \mathrm{PR}} c_{k n t}^{\mathrm{V}, \mathrm{PR}}\right)+ \\
& \sum_{t \in T} \sum_{\omega \in \Omega} \pi_{\omega}\left(\sum_{g \in G}^{\mathrm{C}}\left(C_{g t}^{\mathrm{rup}} r_{g t \omega}^{\mathrm{up}}-C_{g t}^{\mathrm{rdw}} r_{g t \omega}^{\mathrm{dw}}\right)+\right. \\
& \left.\sum_{n \in N} \sum_{k \in K_{t}}\left(C_{k n t}^{\mathrm{V}, \mathrm{rup}} r_{k n t \omega}^{\mathrm{V}, \mathrm{up}}-C_{k n t}^{\mathrm{V}, \mathrm{rdw}} r_{k n t \omega}^{\mathrm{V}, \mathrm{dw}}\right)+\sum_{n \in N} C^{\mathrm{U}}\left(p_{n t \omega}^{\mathrm{U}}+\sum_{c \in C} \tau_{c} p_{n t \omega c}^{\mathrm{U}, \mathrm{PR}}\right)\right)
\end{aligned}
$$


Subject to:

- Day-ahead market constraints

$$
\begin{aligned}
& s_{g t}^{\mathrm{st}} \geq C_{g}^{\mathrm{st}}\left(v_{g t}-v_{g t-1}\right), \quad \forall g \in G^{\mathrm{C}}, \forall t \in T \\
& s_{g t}^{\mathrm{sh}} \geq C_{g}^{\mathrm{sh}}\left(v_{g t-1}-v_{g t}\right), \quad \forall g \in G^{\mathrm{C}}, \forall t \in T \\
& s_{g t}^{\mathrm{st}} \geq 0, s_{g t}^{\mathrm{sh}} \geq 0, \quad \forall g \in G^{\mathrm{C}}, \forall t \in T \\
& P_{\min , g}^{\mathrm{G}} v_{g t} \leq p_{g t}^{\mathrm{DA}} \leq P_{\max , g}^{\mathrm{G}} v_{g t}, \forall g \in G^{\mathrm{C}}, \forall t \in T \\
& p_{g t}^{\mathrm{DA}}-p_{g t-1}^{\mathrm{DA}} \leq P_{\mathrm{up}, g}^{\mathrm{G}} v_{g t-1}+P_{\mathrm{su}, g}^{\mathrm{G}}\left(v_{g t}-v_{g t-1}\right) \\
& +\left(1-v_{g t}\right) P_{\max , g}^{\mathrm{G}}, \quad \forall g \in G^{\mathrm{C}}, \forall t \in T \\
& p_{g t-1}^{\mathrm{DA}}-p_{g t}^{\mathrm{DA}} \leq P_{\mathrm{dw}, g}^{\mathrm{G}} v_{g t}+P_{\mathrm{sd}, g}^{\mathrm{G}}\left(v_{g t-1}-v_{g t}\right) \\
& +\left(1-v_{g t-1}\right) P_{\max , g}^{\mathrm{G}}, \quad \forall g \in G^{\mathrm{C}}, \forall t \in T \\
& p_{g t}^{\mathrm{DA}}+p_{g t}^{\mathrm{S}, \mathrm{DA}}=U_{g t}^{\mathrm{DA}} P_{\max , g}^{\mathrm{G}}, \quad \forall g \in G^{\mathrm{I}}, \forall t \in T \\
& p_{g t}^{\mathrm{DA}} \geq 0, \quad \forall g \in G^{\mathrm{I}}, \forall t \in T \\
& p_{g t}^{\mathrm{S}, \mathrm{DA}} \geq 0, \quad \forall g \in G^{\mathrm{I}}, \forall t \in T \\
& p_{\ell t}^{\mathrm{L}, \mathrm{DA}}=\frac{1}{X_{\ell}}\left(\theta_{O(\ell) t}^{\mathrm{DA}}-\theta_{F(\ell) t}^{\mathrm{DA}}\right), \quad \forall \ell \in L, \forall t \in T \\
& -P_{\max , \ell}^{\mathrm{L}} \leq p_{\ell t}^{\mathrm{L}, \mathrm{DA}} \leq P_{\max , \ell}^{\mathrm{L}}, \quad \forall \ell \in L, \forall t \in T \\
& \sum_{g \in G_{n}} p_{g t}^{\mathrm{DA}}+\sum_{k \in K_{t}}\left(p_{k n t}^{\mathrm{V}, \mathrm{DAD}}-p_{k n t}^{\mathrm{V}, \mathrm{DAC}}\right)-\sum_{\ell \in L_{n}^{\mathrm{O}}} p_{\ell t}^{\mathrm{L}, \mathrm{DA}}+\sum_{\ell \in L_{n}^{\mathrm{F}}} p_{\ell t}^{\mathrm{L}, \mathrm{DA}}=L_{n t}^{\mathrm{DA}},\left(\lambda_{n t}^{\mathrm{DA}}\right) \\
& \forall n \in N, \forall t \in T \\
& 0 \leq c_{g t}^{\mathrm{up}} \leq P_{\max , g}^{\mathrm{G}} v_{g t}, \quad \forall g \in G^{\mathrm{C}}, \forall t \in T \\
& 0 \leq c_{g t}^{\mathrm{dw}} \leq P_{\max , g}^{\mathrm{G}} v_{g t}, \quad \forall g \in G^{\mathrm{C}}, \forall t \in T \\
& c_{k n t}^{\mathrm{V}, \text { up }} \geq 0, \quad \forall k \in K, \forall n \in N, \forall t \in T_{k} \\
& c_{k n t}^{\mathrm{V}, \mathrm{dw}} \geq 0, \quad \forall k \in K, \forall n \in N, \forall t \in T_{k}
\end{aligned}
$$

- Real-time dispatch constraints

$$
\begin{aligned}
& \left\{p_{g t \omega}^{\mathrm{RT}}=p_{g t}^{\mathrm{DA}}+r_{g t \omega}^{\mathrm{up}}-r_{g t \omega}^{\mathrm{dw}}, \quad \forall g \in G^{\mathrm{C}}, \forall t \in T\right. \\
& P_{\mathrm{min}, g}^{\mathrm{G}} v_{g t} \leq p_{g t \omega}^{\mathrm{RT}} \leq P_{\text {max }, g}^{\mathrm{G}} v_{g t}, \quad \forall g \in G^{\mathrm{C}}, \forall t \in T \\
& p_{g t \omega}^{\mathrm{RT}}+p_{g t \omega}^{\mathrm{S}, \mathrm{RT}}=U_{g t \omega}^{\mathrm{RT}} P_{\max , g}^{\mathrm{G}}, \quad \forall g \in G^{\mathrm{I}}, \forall t \in T \\
& p_{g t \omega}^{\mathrm{S}, \mathrm{RT}} \geq 0, \quad \forall g \in G^{\mathrm{I}}, \forall t \in T
\end{aligned}
$$




$$
\begin{aligned}
& p_{g t \omega}^{\mathrm{RT}}-p_{g t-1, \omega}^{\mathrm{RT}} \leq P_{\mathrm{up}, g}^{\mathrm{G}} v_{g t-1}+P_{\mathrm{st}, g}^{\mathrm{G}}\left(v_{g t}-v_{g t-1}\right) \\
& +\left(1-v_{g t}\right) P_{\text {max }, g}^{\mathrm{G}}, \forall g \in G^{\mathrm{C}}, \forall t \in T \\
& p_{g t-1, \omega}^{\mathrm{RT}}-p_{g t \omega}^{\mathrm{RT}} \leq P_{\mathrm{dw}, g}^{\mathrm{G}} v_{g t}+P_{\mathrm{sh}, g}^{\mathrm{G}}\left(v_{g t-1}-v_{g t}\right) \\
& +\left(1-v_{g t-1}\right) P_{\max , g}^{\mathrm{G}}, \forall g \in G^{\mathrm{C}}, \forall t \in T \\
& 0 \leq r_{g t \omega}^{\text {up }} \leq c_{g t}^{\text {up }}, \quad \forall g \in G^{\mathrm{C}}, \forall t \in T \\
& 0 \leq r_{g t \omega}^{\mathrm{dw}} \leq c_{g t}^{\mathrm{dw}}, \quad \forall g \in G^{\mathrm{C}}, \forall t \in T \\
& 0 \leq r_{k n t \omega}^{\mathrm{V} \text {,up }} \leq c_{k n t}^{\mathrm{V} \text {,up }}, \quad \forall k \in K, \forall n \in N, \forall t \in T \\
& 0 \leq r_{k n t \omega}^{\mathrm{V}, \mathrm{dw}} \leq c_{k n t}^{\mathrm{V}, \mathrm{dw}}, \quad \forall k \in K, \forall n \in N, \forall t \in T \\
& p_{\ell t \omega}^{\mathrm{L}, \mathrm{RT}}=\frac{1}{X_{\ell}}\left(\theta_{O(\ell) t \omega}^{\mathrm{RT}}-\theta_{F(\ell) t \omega}^{\mathrm{RT}}\right), \quad \forall \ell \in L, \forall t \in T \\
& -P_{\max , \ell}^{\mathrm{L}} \leq p_{\ell t \omega}^{\mathrm{L}, \mathrm{RT}} \leq P_{\max , \ell}^{\mathrm{L}}, \quad \forall \ell \in L, \forall t \in T \\
& \sum_{g \in G_{n}^{\mathrm{C}}}\left(r_{g t \omega}^{\mathrm{up}}-r_{g t \omega}^{\mathrm{dw}}\right)+\sum_{g \in G_{n}^{\mathrm{I}}}\left(p_{g t \omega}^{\mathrm{RT}}-p_{g t}^{\mathrm{DA}}\right)+\sum_{k \in K_{n}}\left(r_{k n t \omega}^{\mathrm{V}, \mathrm{up}}-r_{k n t \omega}^{\mathrm{V}, \mathrm{dw}}\right)- \\
& \sum_{\ell \in L_{n}^{\mathrm{O}}}\left(p_{\ell t \omega}^{\mathrm{L}, \mathrm{RT}}-p_{\ell t}^{\mathrm{L}, \mathrm{DA}}\right)+\sum_{\ell \in L_{n}^{\mathrm{F}}}\left(p_{\ell t \omega}^{\mathrm{L}, \mathrm{RT}}-p_{\ell t}^{\mathrm{L}, \mathrm{DA}}\right)+p_{n t \omega}^{\mathrm{U}}=L_{n t \omega}^{\mathrm{RT}}-L_{n t}^{\mathrm{DA}},\left(\lambda_{n t \omega}^{\mathrm{RT}}\right) \\
& \forall n \in N, \forall t \in T \\
& 0 \leq p_{n t \omega}^{\mathrm{U}} \leq L_{n t \omega}^{\mathrm{RT}}, \quad \forall n \in N, \forall t \in T \\
& 0 \leq p_{g t \omega c}^{\mathrm{PR}} \leq-\frac{1}{R_{g}} \Delta f_{t \omega c}, \forall g \in G^{\mathrm{C}}, \forall t \in T, \forall c \in C, \text { if } V_{g c}^{\mathrm{post}}=1 \\
& p_{g t \omega c}^{\mathrm{PR}}+p_{g t \omega}^{\mathrm{RT}}=0, \forall g \in G^{\mathrm{C}}, \forall t \in T, \forall c \in C, \text { if } V_{g c}^{\mathrm{post}}=0 \\
& V_{g c}^{\mathrm{post}}\left(p_{g t \omega}^{\mathrm{RT}}+p_{g t \omega c}^{\mathrm{PR}}\right) \leq P_{\text {max }, g}^{\mathrm{G}} v_{g t}, \forall g \in G^{\mathrm{C}}, \forall t \in T, \forall c \in C \\
& 0 \leq p_{k n t \omega c}^{\mathrm{V}, \mathrm{PR}} \leq c_{k n t}^{\mathrm{V}, \mathrm{PR}}, \forall k \in K, \forall n \in N, \forall t \in T_{k}, \forall c \in C \\
& 0 \leq p_{k n t w c}^{\mathrm{V}, \mathrm{PR}} \leq-\frac{1}{R^{\mathrm{V}}} \Delta f_{t \omega c}, \quad \forall k \in K, \forall n \in N, \forall t \in T_{k}, \forall c \in C \\
& -\Delta f_{\max } \leq \Delta f_{t \omega c} \leq \Delta f_{\max }, \forall t \in T, \forall c \in C \\
& \sum_{g \in G} p_{g t \omega c}^{\mathrm{PR}}+\sum_{k \in K_{t}} p_{k n t \omega c}^{\mathrm{V}, \mathrm{PR}}-p_{n t \omega c}^{\mathrm{U}, \mathrm{PR}}=0, \forall t \in T, \forall n \in N, \forall c \in C \\
& 0 \leq p_{n t \omega c}^{\mathrm{U}, \mathrm{PR}} \leq L_{n t \omega}^{\mathrm{RT}}-p_{n t \omega}^{\mathrm{U}}, \forall n \in N, \forall t \in T, \forall c \in C \\
& \operatorname{soc}_{k n t w c}=N_{k n}^{\mathrm{V}} S O C_{k n}^{0}, \forall k \in K, \forall n \in N, t=t_{k}^{0}-1, \forall c \in C \\
& \operatorname{soc}_{k n t \omega c} \geq N_{k n}^{\mathrm{V}} S O C_{k n}^{\mathrm{F}}, \forall k \in K, \forall n \in N, t=t_{k}^{\mathrm{F}}, \forall c \in C \\
& \operatorname{soc}_{k n t \omega c}=\operatorname{soc}_{k n t-1, \omega 0}+\alpha_{k} e_{k n t \omega c}^{\mathrm{C}}-\frac{1}{\alpha_{k}} e_{k n t \omega c}^{\mathrm{D}}, \\
& \forall k \in K, \forall n \in N, t \in T_{k}, \forall c \in C \\
& N_{k n}^{\mathrm{V}} S O C_{\min , k} \leq \operatorname{soc}_{k n t \omega c} \leq N_{k n}^{\mathrm{V}} S O C_{\max , k}, \\
& \forall k \in K, \forall n \in N, t \in T_{k}, \forall c \in C
\end{aligned}
$$




$$
\begin{aligned}
& e_{k n t \omega c}^{\mathrm{C}}=p_{k n t}^{\mathrm{V}, \mathrm{DAC}}-r_{k n t \omega}^{\mathrm{V}, \mathrm{upC}}+r_{k n t \omega}^{\mathrm{V}, \mathrm{dwC}}-D^{\mathrm{PR}} p_{k n t \omega c}^{\mathrm{V}, \mathrm{PRC}} \text {, } \\
& \forall k \in K, \forall n \in N, \forall t \in T_{k}, \forall c \in C \\
& e_{k n t \omega c}^{\mathrm{D}}=p_{k n t}^{\mathrm{V}, \mathrm{DAD}}+r_{k n t \omega}^{\mathrm{V}, \mathrm{upD}}-r_{k n t \omega}^{\mathrm{V}, \mathrm{dwD}}+D^{\mathrm{PR}} p_{k n t \omega c}^{\mathrm{V}, \mathrm{PRD}} \text {, } \\
& \forall k \in K, \forall n \in N, \forall t \in T_{k}, \forall c \in C \\
& 0 \leq p_{k n t}^{\mathrm{V}, \mathrm{DAC}}-r_{k n t \omega}^{\mathrm{V}, \mathrm{upC}}+r_{k n t \omega}^{\mathrm{V}, \mathrm{dwC}}-p_{k n t \omega c}^{\mathrm{V}, \mathrm{PRC}} \leq N_{k n}^{\mathrm{V}} P_{\max }^{\mathrm{V}} \text {, } \\
& \forall k \in K, \forall n \in N, \forall t \in T_{k}, \forall c \in C \\
& 0 \leq p_{k n t}^{\mathrm{V}, \mathrm{DAD}}+r_{k n t \omega}^{\mathrm{V}, \mathrm{upD}}-r_{k n t \omega}^{\mathrm{V}, \mathrm{dwD}}+p_{k n t \omega c}^{\mathrm{V}, \mathrm{PRD}} \leq N_{k n}^{\mathrm{V}} P_{\text {max }}^{\mathrm{V}} \text {, } \\
& \forall k \in K, \forall n \in N, \forall t \in T_{k}, \forall c \in C \\
& r_{k n t \omega}^{\mathrm{V}, \mathrm{up}}=r_{k n t \omega}^{\mathrm{V}, \mathrm{upC}}+r_{k n t \omega}^{\mathrm{V}, \mathrm{upD}}, \forall k \in K, \forall n \in N, \forall t \in T_{k} \\
& 0 \leq r_{k n t \omega}^{\mathrm{V}, \text { upC }} \leq p_{k n t}^{\mathrm{V}, \mathrm{DAC}}, \forall k \in K, \forall n \in N, \forall t \in T_{k} \\
& 0 \leq r_{k n t \omega}^{\mathrm{V}, \text { upD }} \leq N_{k n}^{\mathrm{V}} P_{\text {max }}^{\mathrm{V}}-p_{k n t}^{\mathrm{V}, \mathrm{DAD}}, \forall k \in K, \forall n \in N, \forall t \in T_{k} \\
& r_{k n t \omega}^{\mathrm{V}, \mathrm{dw}}=r_{k n t \omega}^{\mathrm{V}, \mathrm{dwC}}+r_{k n t \omega}^{\mathrm{V}, \mathrm{dwD}}, \forall k \in K, \forall n \in N, \forall t \in T_{k} \\
& 0 \leq r_{k n t \omega}^{\mathrm{V}, \mathrm{dwC}} \leq N_{k n}^{\mathrm{V}} P_{\max }^{\mathrm{V}}-p_{k n t}^{\mathrm{V}, \mathrm{DAC}}, \forall k \in K, \forall n \in N, \forall t \in T_{k} \\
& 0 \leq r_{k n t \omega}^{\mathrm{V}, \mathrm{dwD}} \leq p_{k n t}^{\mathrm{V}, \mathrm{DAD}}, \forall k \in K, \forall n \in N, \forall t \in T_{k} \\
& p_{k n t \omega c}^{\mathrm{V}, \mathrm{PR}}=p_{k n t \omega c}^{\mathrm{V}, \mathrm{PRC}}+p_{k n t \omega c}^{\mathrm{V}, \mathrm{PRD}}, \forall k \in K, \forall n \in N, \forall t \in T_{k}, \forall c \in C \\
& \}, \forall \omega \in \Omega
\end{aligned}
$$

where $\Theta$ is the set of all optimization variables in problem (B.2)-(B.54).

The objective function (B.2) formulates the total expected operating cost considering the day-ahead and real-time energy markets, the reserve capacity market and the frequency regulation service provided by the PEVs. This objective function is subject to two sets of constraints. The first group of constraints, (B.2)-(B.17), are used to formulate the day-ahead market, while constraints (B.18)-(B.54) model the real-time dispatch for each considered scenario $\omega \in \Omega$. Constraints (B.2)-(B.4) formulate startup and shutdown costs of generating units. The power limits and ramps of the energy traded in the day-ahead energy market by dispatchable generating units are formulated through constraints (B.5)-(B.7). The power limits of intermittent units are established by constraints (B.8)-(B.10). The upper limit of the power output of intermittent units is equal to the capacity of the unit times an availability factor, $U_{g t}^{\mathrm{DA}}$, that indicates the expected availability of unit $g$ in period $t$. The power flows in the transmission lines are modeled by constraints (B.11)-(B.12). The power balance in the day-ahead energy market is established by (B.13). Constraints (B.14)-(B.17) enforce the bounds for the up and down reserve capacity provided by generating units and PEVs. Observe that the formulation of minimum up and down times could also be included in the set of technical constraints of generating units, as done in [38]. However, due to space limitation and that these constraints are usually not enforced in small-size generating units, as those included in isolated power systems, the explicit formulation of these constraints has been omitted here. 
The power limits of generating units in the real-time dispatch are formulated by (B.18)(B.21). The power ramps of generating units in the real-time dispatch are formulated by (B.22)-(B.23). The reserve energy deployments of generating units and PEVs are bounded by the reserve capacities in (B.24)-(B.27). The resulting transmission power flows in the real-time dispatch are modeled by constraints (B.28)-(B.29). The power balance in the real-time dispatch is enforced by constraint (B.30). Constraints (B.30) limit the value of the unserved demand in the real-time dispatch.

The primary frequency response is formulated by constraints (B.32)-(B.39). Unexpected failures of generating units are defined as contingencies which happen in the post-contingency states $c \in C$. The binary parameter $V_{g c}^{\text {post }}$ is used to characterize whether a unit suffers a failure or not in post-contingency state $c$. In this manner, if $V_{g c}^{\text {post }}=1$ it is considered that unit $g$ is available in period $t$, whereas $V_{g c}^{\text {post }}=0$ indicates that unit $g$ suffers an equipment failure and its power output in period $t$ will be null. Index $c=0$ is used to denote the post-contingency state in which there does not exist any failure and all units are available. The variation of the power output in each dispatchable generating unit $g$ in period $t$ and scenario $\omega$ and post-contingency state $c$ is modeled by variable $p_{g t \omega c}^{\mathrm{PR}}$. In this manner, the increment of the power output of all committed units that do not suffer contingencies will be always positive and it will be bounded by either the droop of the unit or its capacity (constraints (B.32) and (B.34)). However, if a unit suffers a contingency, its power output in the post-contingency state will decrease a value equal to its power output in the real-time dispatch $p_{g t \omega}^{\mathrm{RT}}$ as stated in constraints (B.33). Constraints (B.35) and (B.36) model the participation of PEVs in PFR, which is bounded by the PFR capacity settled in the day-ahead market and the droop control. The post-contingency frequency deviation from the nominal value is constrained in (B.37). The power balance in the post-contingency state is formulated in (B.38). Constraints (B.39) limit the unserved-demand in the post-contingency state.

Finally, the state-of-charge constraints of PEVs are formulated by (B.40)-(B.54). Constraints (B.40)-(B.41) establish the status of the batteries of PEVs at the beginning and the end of the charging periods, respectively. The energy stored by PEVs in each hour $t$ is computed by constraint (B.42) as a function of the energy stored in the previous hour, $t-1$, plus the energy charged from the power grid and minus the energy discharged from PEVs. The lower and upper bounds of the energy stored in the batteries are established through constraints (B.43). The actual values of energy charged from and discharged to the grid in each bus, period, scenario and contingency considering the participation in the day-ahead energy market, the deployment of up and down reserves and the provision of PFR after a contingency are formulated through constraints (B.44) and (B.45). Note that the power charged and discharged 
for PFR is multiplied by factor $D^{\mathrm{PR}}$ in order to compute the energy associated with this service. This is because the maximum duration allowed for PFR, $D^{\mathrm{PR}}$, is less than one hour. The upper and lower bounds to the power charged from and discharged to the grid are established by constraints (B.46) and (B.47). The deployment of up reserves is formulated by constraints (B.48)-(B.50). Observe that up reserve $r_{k n t \omega c}^{\mathrm{V} \text {,up }}$ can be provided by PEVs either increasing the discharge, $r_{k n t \omega c}^{\mathrm{V} \text {,upD }}$, or decreasing the charge, $r_{k n t \omega c}^{\mathrm{V} \text {,upC }}$. A similar reasoning can be made for the provision of down reserve (constraints (B.51)-(B.53)) and PFR (constraints (B.54)). 